Preprint, January 14, 2022

\title{
Modelling Consensus Emergence With Nonlinear Dynamics
}

\author{
Yvette Baurne, \\ Department of Statistics, \\ Lund University \\ Frédéric Delmar, \\ Department of Entrepreneurship and Innovation, \\ emlyon Business School, \\ Jonas Wallin \\ Department of Statistics, \\ Lund University
}

\begin{abstract}
The study of emergent, bottom-up, processes has long been of interest within organizational and group research. Emergent processes refer to how dynamic interactions among lower-level units (e.g. individuals) over time form a new, shared, construct or phenomena at a higher level (e.g. work group). To properly study emergence of shared constructs one needs models, and data, that both take into account variability across individuals and groups (multilevel), and variability over time (longitudinal). This article makes three contribution to the modelling and theory of of consensus emergence. First, we formulate two separate patterns of consensus emergence; homogeneous and heterogeneous. Homogeneous consensus emergence is characterised by gradual and almost deterministic adjustments of the individual trajectories, whereas heterogeneous consensus emergence show more randomly oscillating trajectories towards consensus. Second, we introduce a model-invariant statistic that measures the strength of the consensus; and allows for comparisons between different models and patterns of consensus emergence. Third, we show how Gaussian Processes can be used to further extend the consensus emergence models, allowing them to capture nonlinear dynamics, on both individual and group level, in emergent processes. Using an established data set, we show that conclusions on the pattern of consensus emergence can change depending on whether the nonlinear group mean change over time is adequately modelled or not. Thus it is crucial to correctly capture the group dynamics to properly understand the consensus emergence.
\end{abstract}

*yvette.baurne@stat.lu.se

†delmar@em-lyon.com, ORCID: 0000-0003-4974-9031

¥jonas.wallin@stat.lu.se 


\section{Introduction}

Research on emergent processes has a long tradition in the social sciences and in the study of organizations and groups (Baronchelli, 2018; Kozlowski et al., 2013; Wee \& Taylor, 2018). Emergent processes refer to how the interactions between lower-level (e.g. individual level) elements unfold over time to yield a structure or collective phenomena at a higher level (Bedau, 1997; Fulmer \& Ostroff, 2016, Kauffman \& Clayton, 2006, Larson et al., 2020). In group research, this would mean how the interactions among individuals in a group (bottom-up) generate higher level emergent states. Such emergent states are collective phenomena that affect how individuals then behave in the group (top-down) (Marks et al., 2001; Rapp et al., 2021). Emergent processes are bottom-up where:

- a new higher-level "whole" is formed from the individual "parts";

- involving dynamic interactions among the individual elements, eventually coalescing around an inflection point at a higher level of analysis (i.e. convergence or consensus);

- allowing the new state or form, as a collective, to guide future actions, behaviors or activities (i.e., emergence);

- is a longitudinal process that occurs over time.

Research has identified a number of different emergent processes and a dominant process is the emergence of consensus. Emergent consensus involves an explanation of how meanings and understanding are shared, learned and adapted over time within a social group through everyday interactions, becoming embodied in the system through self-reproduction (Thornton et al., 2012). These emergent processes such as the emergence of group-level phenomena involve changes happening over time.

Consequently, research on emergence and related group processes necessitates longitudinal research (Fulmer \& Ostroff, 2016; Kozlowski \& Chao, 2018; Wang et al., 2016). Such research is of fundamental importance to understand and support group level processes over time, which due to individual and group relation are multilevel. Lang et al. (2018) argue that the lack of advancement in understanding how group behavior emerges over time as bottom-up processes is partly due to the lack of appropriate statistical methodological tools.

Data on consensus emergence are by necessity both longitudinal and multilevel. Such data have specific properties and challenges that need to be addressed in order to correctly analyze them. For instance the observations are often highly dependent in complicated ways. Specifically, different emergence patterns (different multilevel models) will lead to different correlation structures between observations (Diggle, 1988; Munoz et al., 1992). In Asar et al., 2020 it is shown that finding the correct correlation structure of the data is crucial for correct statistical analysis. The inability to correctly analyze such correlation structures can lead to incorrect conclusions on how group processes emerge and if they emerge at all. To extract these correlation structures from data the main tools are the multilevel models (Goldstein, 2011; Humphrey \& LeBreton, 2019, Little, 2013). Recent works point out that these types of patterns can be studied within a heterogeneous variance (multilevel) model context (Lang, Bliese, \& Adler, 2019; Lester et al., 2021) where the consensus emergence is captured as changes in the error term variance over time, i.e. heteroscedasticity. In this context the heteroscedasticity over time is not viewed as a violation of assumptions, as common for the multilevel growth model (Bliese \& Ployhart, 2002), but as a way to gain insights into how group processes emerge. 
To determine existence of an emergence pattern in the multilevel models, the researcher often extract some statistic and tests it significance, e.g., a correlation parameter. An issue when studying the emergence is that the classical statistics poorly captures the dynamics of consensus emergence. Among the most commonly used statistics for consensus emergence are the Intraclass Correlation Coefficients (ICCs), used to measure both interrater (agreement within the group) and intrarater (agreement of the individual over repeated measures) reliability in groups, and measures of within-group agreement such as $r_{w g}$ (Allen \& O'Neill, 2015, Newman \& Sin, 2020 Wittmer \& LeBreton, 2021). See for example Allen and O'Neill (2015) who reports measures of shared grouplevel constructs from 109 studies in organizational settings during the years 1991-2010, which all use at least one measure of ICC or within-group agreement. In a recent series of articles, Lang with colleagues (Lang et al., 2018, Lang, Bliese, \& Runge, 2019) raise criticism over the ICC to estimate consensus emergence, especially because ICC cannot distinguish group level change from individual level change over time. Similar to Lang et al. (2018), we show how the usage of ICC for measuring consensus emergence can conflate group effects with consensus emergence effects. We prove in a constructed example that it is not possible to separate an increasing variation between groups from consensus emergence (which is within the group) using ICC (See Figure 1). Although the example is theoretical and extreme it shows the limitation with ICC for the estimation of consensus emergence. The main limit is that ICC lacks the temporal component that is fundamental for emergence to happen (Cronin et al., 2011; Humphrey \& Aime, 2014 Kozlowski et al., 2013). Thus to measure consensus emergence, we introduce a new measure usable both empirically (based on raw data and no model assumption) and in a model.

Our contribution is threefold. First, we identify two different patterns of consensus emergence and thus define two types of consensus emergence (multilevel) models, which we denote Homogeneous and Heterogeneous Consensus Emergence Model, HomCEM and HetCEM, respectively. The two models differ in that the HomCEM has a gradual and almost deterministic adjustment of the individual trajectory to consensus, whereas HetCEM has a more randomly oscillating trajectory to consensus. The Consensus Emergence Model (CEM) introduced in (Lang et al., 2018) is a Heterogeneous consensus emergence model.

Second, because we identify different patterns of consensus emergence, we need a common mathematical definition of what consensus emergence is in multilevel models. We use this definition to construct a statistic, $r(t)$, to measure the strength of consensus. Simply put the statistic indicates how much an individuals variability has decreased over time with respect to the initial time observation. This statistic does not suffer from the same shortcomings as ICC does. It is also not model specific so different models can be compared. One can also get a, biased, estimate of the statistic directly from data without any model assumptions.

Third, to handle the complex dynamics existing in emergent processes we show how to use Gaussian processes (Diggle, 1988, Rasmussen, 2003) in multilevel consensus emergence models. The Gaussian processes can capture nonlinear time dynamics that can be hard to find using the classical, linear, multilevel approach. Correctly capturing these complexities is crucial to decipher which type of consensus emergence is present. This is illustrated with the Sherif data, where among the linear consensus emergence models the most likely pattern is heterogeneous. After including models with non-linear group dynamics, Gaussian processes, the most likely pattern is then homogeneous. The reason for this change is that the simpler linear consensus emergence models fail to correctly capture the correlation structure.

We structure the paper as follow. In section 2 we have a general discussion of consensus emergence and mathematical definition. Particularly, we introduce two general patterns for consensus 
emergence, heterogeneous and homogeneous. In section 3, we introduce the multilevel model which is the structure of consensus emergence models studied here. In section 4, we discuss statistics for consensus emergence. In particular, we show mathematically why ICC is inadequate to measure consensus emergence. We introduce a new statistic to measure the effect of consensus emergence. This is needed to compare the magnitude of consensus for the different models that are studied in the paper. In section 5, we show how to incorporate heterogeneous and homogeneous consensus emergence in a multilevel model. In section 6, we show how to use Gaussian Processes in consensus emergence models. In section 7 , we apply our models to field and simulated data. In section 8 , we discuss the implication of our findings.

\section{Consensus emergence}

How team members move towards consensus is important to our ability to detect the emergence of consensus. We give two examples which describe two different types or patterns of consensus emergence: heterogeneous and homogeneous consensus emergence. Our account is somewhat different from a cross-sectional account of team variance as described by DeRue et al. (2010). For both types of consensus, if there is emergence, the team variance structure at the first measurement point will gradually disappear, but their pattern of disappearance will differ. Both types of consensus emergence fits definition 1 below. The two examples are illustrated in Figure 1. In both examples, we have a work team of four people recently put together. We are interested in the workplace climate. We ask them to rate the workplace climate on four occasions, each one month apart. The initial measurements on the workplace climate are varying a lot. At the second measurement occasion, we see that they are moving closer to each other, at the third measurement even closer, to finally reach consensus at the fourth measurement. After working together in a group for some time, the individuals are getting more aligned in their ratings on the climate. 


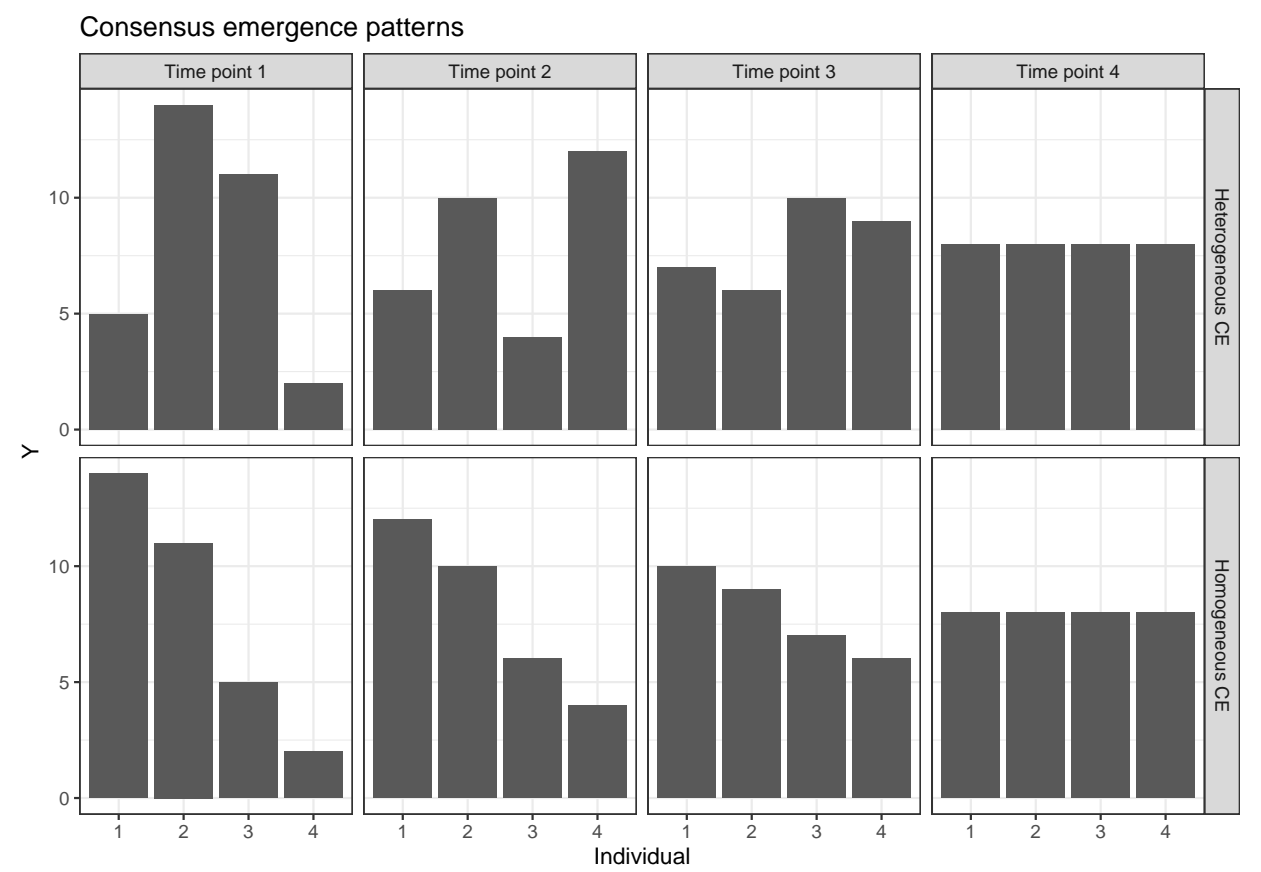

Figure 1: Two examples of different consensus emergence patterns.

Heterogeneous consensus emergence. The top row in Figure 1 illustrates an example of heterogeneous consensus emergence. Here, each individual changes over time to be either below or above the group mean. Further, the magnitude of the differences between the individuals in the team decreases over time which results in consensus emergence. This would be the case of a team processing ambiguous information where individuals are reaching consensus, but before that they can change their perceptions with great magnitude between measurement occasions. This creates a pattern of individual measurements that oscillates freely above and below the group mean.

Homogeneous consensus emergence. The bottom row in Figure 1 illustrates an example of homogeneous consensus emergence. The individual measurements remain, on the four occasions, on the same side of the group mean. The individual trajectories converges towards the group mean resulting in consensus emergence. This leads to an almost deterministic pattern compared to the more random pattern of the heterogeneous consensus emergence.

To incorporate the two types of consensus emergence and to measure the consensus emergence effect in a statistical model, we need to have an exact mathematical definition of the concept consensus emergence. This since there exists many different ways in which consensus emergence can enter a model and hence it can be difficult to compare the level of consensus emergence for two different models. By introducing an exact definition we can explore how the concepts work for a wide range of models and we will do so later in this article.

We view consensus emergence as a within-group effect. Hence, we are examining the increase (or decrease) in similarity between two or more individuals within a group over time. Let $p_{i t}$ denote a measurement of interest for individual $i$ at time point $t$. For two time points $t_{0}<t_{1}$ the similarity of two individuals, $i$ and $k$, has increased if $\left|p_{i t_{0}}-p_{k t_{0}}\right|>\left|p_{i t_{1}}-p_{k t_{1}}\right|$ (and decreased if 
$\left.\left|p_{i t_{0}}-p_{k t_{0}}\right|<\left|p_{i t_{1}}-p_{k t_{1}}\right|\right)$.

Our formal definition of consensus emergence in a multilevel setting is as follows.

Definition 1. Let $P_{i j}(t)$ denote a quantity of interest for individual $i=1, \ldots, n_{j}$ in group $j$, at time $t$. There is consensus emergence in group $j$ between the time points $t_{0}<t_{1}$ for the quantity if, for two uniformly randomly selected individuals $i, k$,

$$
\mathbb{E}\left[\left(P_{i j}\left(t_{0}\right)-P_{k j}\left(t_{0}\right)\right)^{2}\right]>\mathbb{E}\left[\left(P_{i j}\left(t_{1}\right)-P_{k j}\left(t_{1}\right)\right)^{2}\right] .
$$

We choose this definition because it only includes differences between individuals in the same group, causing the effect of the group mean to disappear. This is an important property because it separates (linear) individual and group effects which is crucial in modelling consensus emergence. As we detail later, ICC conflates these two effects. This is the reason to why we should not use ICC to assess consensus emergence.

\section{Multilevel models}

We study consensus emergence in a multilevel setting. Here we introduce the necessary multilevel theory. First is the two-level model which goes by various names, e.g., the intercept-only multilevel model or variance components model (Goldstein, 2011; Lang \& Bliese, 2019). The two levels, in organization or group research, represent a group level (here denoted $G$ ) and an observation/individual level (here denoted $P$ ). Here we cannot separate measurement variability and individual (personal) variability. If one has repeated measurements from the same individual one can separate this variability by a three level model. The three levels are then a group level, denoted $G$, an individual/person level, denoted $P$, and a measurement level, denoted $Y$.

In more detail we define the two-level model as follows: The response $P_{i j}$ is a function of a group mean $G_{j}$, which in turn consists of an overall mean $G_{0}$ (the intercept) and group-specific deviations from that mean $e_{j}^{G}$, and the error term $e_{i j}^{P}$ :

$$
\begin{aligned}
\text { Level } 1 \text { (person - measurement): } P_{i j} & =G_{j}+e_{i j}^{P}, \\
\text { Level } 2 \text { (group) }: G_{j} & =G_{0}+e_{j}^{G} .
\end{aligned}
$$

Typically it is assumed that error terms are independent and $e_{i j}^{P} \sim N\left(0, \sigma_{P}^{2}\right)$, and $e_{j}^{G} \sim N\left(0, \sigma_{G}^{2}\right)$. The model allow us to partition the total variance $\left(\sigma_{P}^{2}+\sigma_{G}^{2}\right)$ of the data into separate parts originating from level 1 (person-measurement level) and level 2 (group level), $\sigma_{P}^{2}$ and $\sigma_{G}^{2}$, respectively. When we have repeated measurements we can expand into a three-level model which allows for separation of individual and measurement variability.

For the three-level model the person-measurement level from the two-level model is split into two parts: a measurement level (denoted $Y_{t i j}$ ) and a person-specific level (denoted $P_{i j}$ ):

$$
\begin{aligned}
\text { Level } 1 \text { (measurement): } Y_{t i j} & =P_{i j}+e_{t i j}^{Y}, \\
\text { Level } 2 \text { (person): } P_{i j} & =G_{j}+e_{i j}^{P}, \\
\text { Level } 3 \text { (group): } G_{j} & =G_{0}+e_{j}^{G} .
\end{aligned}
$$

Typically, error terms are assumed independent and $e_{t i j}^{Y} \sim N\left(0, \sigma_{Y}^{2}\right), e_{i j}^{P} \sim N\left(0, \sigma_{P}^{2}\right)$, and $e_{j}^{G} \sim$ $N\left(0, \sigma_{G}^{2}\right)$. In addition to the partitioning for the two-level model, it also includes person-specific 
deviations, $e_{i j}^{P}$, from the group mean. Thus the total variance is partitioned into $\sigma_{Y}^{2}, \sigma_{P}^{2}$, and $\sigma_{G}^{2}$ (measurement, person and group, respectively).

The multilevel model specification allows for modeling systematic changes in error variances on all levels. Here we show two examples of how to add a systematic changes in the error variances over time for individual level and group level.

Example 1. Here, we introduce individual variability over time. We do this by changing the individual errors (Level 2) in (2) over time and thus we need to add an index $t$ for time points. Let $e_{t i j}^{P} \sim N\left(0, \sigma_{P}^{2} \exp \left[2 \delta_{P} t\right]\right)$. The variance is a function of time and the parameter $\delta_{P}$, which controls the rate of change over time. The exponent ensures that the variance is always positive.

$$
\begin{aligned}
\text { Level } 1 \text { (measurement): } Y_{t i j} & =P_{t i j}+e_{t i j}^{Y}, \\
\text { Level 2 (person): } P_{t i j} & =G_{j}+e_{t i j}^{P}, \\
\text { Level 2 (group): } G_{j} & =G_{0}+e_{j}^{G},
\end{aligned}
$$

where $e_{t i j}^{P} \sim N\left(0, \sigma_{P}^{2} \exp \left[2 \delta_{P} t\right]\right)$, and $e_{j}^{G} \sim N\left(0, \sigma_{G}^{2}\right)$.

Example 2. Next, we introduce group variability over time. We do this by letting the group errors (Level 3) in (2) change over time, multiplying the residual $e_{j}^{G}$ with $\exp \left[\delta_{G} t\right]$. The parameter $\delta_{G}$ controls the rate of change over time. Note that the notation with subscript $t$ indicates that the function of time is discontinuous, while the notation with $(t)$ indicates that the function is continuous.

$$
\begin{aligned}
\text { Level 1 (measurement): } Y_{t i j} & =P_{t i j}+e_{t i j}^{Y}, \\
\text { Level 2 (person): } P_{i j}(t) & =G_{j}(t)+e_{i j}^{P}, \\
\text { Level 2 (group): } G_{j}(t) & =G_{0}+\exp \left[\delta_{G} t\right] e_{j}^{G},
\end{aligned}
$$

where $e_{i j}^{P} \sim N\left(0, \sigma_{P}^{2}\right)$, and $e_{j}^{G} \sim N\left(0, \sigma_{G}^{2}\right)$.

For the first example the person variance changes over time. For the second example the group mean changes over time. Note that different patterns of change in the error variances are implied by Example 1 and 2. In Example 1, the error belongs to a new Normal distribution at each time point $t$. The mean is always zero but the variance is increasing or decreasing depending on the value of $\delta_{P}$. This enables the error for an individual $i$ to alternate between values above and below the group mean $G_{j}$ as $t$ increases, much like the pattern of heterogeneous consensus emergence. In Example 2, the variance of $e_{i j}^{P}$ is independent of time, it is the same for all time points. The group level error, $\exp \left[\delta_{G} t\right] e_{j}^{G}$, does however change as a function of time. Here the parameter $\delta_{G}$ controls the rate of change. Because the exponent cannot take negative values, the sign of the error function will always be the same for a group $j$ over time. This means that if $G_{j}\left(t_{0}\right)>G_{0}$ then $G_{j}(t)>G_{0}$ for all $t$. That is, if the group mean is larger than the overall mean $G_{0}$ at the initial time point, it will be larger than the overall mean for all time points. The reverse holds for group means smaller than the overall mean $G_{0}$. This pattern of group error variance change over time resembles the pattern that one would expect for individuals exhibiting homogeneous consensus emergence.

In Section 5, we use the methods introduced in these two examples to build different types of consensus emergence for multilevel models. These will be the two main tools for estimating either homogeneous consensus emergence or heterogeneous consensus emergence. 


\section{Statistics for consensus emergence}

There exists a broad range of statistics to measure shared group-level constructs within applied research, e.g. the Intraclass Correlation Coefficient (ICC) and the within-group agreement measure $r_{w g}$. These methods are widely spread and used across many contexts. Their versatility comes with a drawback: it is often unclear how well they apply in a specific context. For instance, there is no natural way to incorporate changing behavior over time, which is fundamental for consensus emergence defined as a change in behavior over time.

We demonstrate the problem with the most commonly used statistic ICC as a measure of consensus emergence and then introduce our proposed measure of consensus emergence: $r(t)$.

The problem caused by not taking time into account is the conflation of between-group and within-group variance. For many of the statistics used for consensus emergence, which are crosssectional, when comparing values over time it is not possible to derive whether a change originates from a change in within-group variance or between-group variance.

We use an example to illustrate this conflation problem. We use the two-level model 11 and add the variance function from Example 1 to the person/measurement level, and add the variance function from example 2 to the group level. Then we have a model in which we can control for changes in both within- and between-group variance. That is, the model is

$$
\text { Level } 1 \text { (person/measurement): } \begin{aligned}
P_{t i j} & =G_{j}(t)+e_{t i j}^{P}, \\
\text { Level } 2 \text { (group): } G_{j}(t) & =G_{0}+\exp \left[\delta_{G} t\right] e_{j}^{G},
\end{aligned}
$$

where $e_{t i j}^{P} \sim N\left(0, \sigma_{P}^{2} \exp \left[2 \delta_{P} t\right]\right)$, and $e_{j}^{G} \sim N\left(0, \sigma_{G}^{2}\right)$. Here $\exp \left[2 \delta_{P} t\right]$ controls the within-group variance, where a negative $\delta_{P}$ gives a consensus emergence behavior, i.e., a decreasing withingroup variance over time, and $\exp \left[2 \delta_{G} t\right]$ controls the between-group variance, a positive $\delta_{G}$ gives an increasing between-group variance over time. Next, we show that the ICC will have a problem separating these two variances.

\subsection{ICC as a measure of consensus emergence}

ICC gives the correlation between two individuals within a group. For the two-level model (1) the ICC is

$$
I C C=\frac{\sigma_{G}^{2}}{\sigma_{G}^{2}+\sigma_{P}^{2}} .
$$

We give the general definition of ICC in Appendix A.

The higher the similarity between individuals, the higher the ICC will be, which then should imply more consensus. However, as ICC is a cross-sectional measure and thus does not capture the time aspect of consensus emergence, there will be conflation of variances when we compare values over time. In particular, an increased ICC value over time can be caused by:

- an increased similarity between individuals within a group, i.e., a decrease in within-group variance (true consensus emergence), or

- a decreased similarity between groups, i.e., an increase in between-group variation.

To illustrate this conflation problem when using ICC, we generate two data sets from model 5 described above. Each data set consists of four groups with four individuals in each group, 

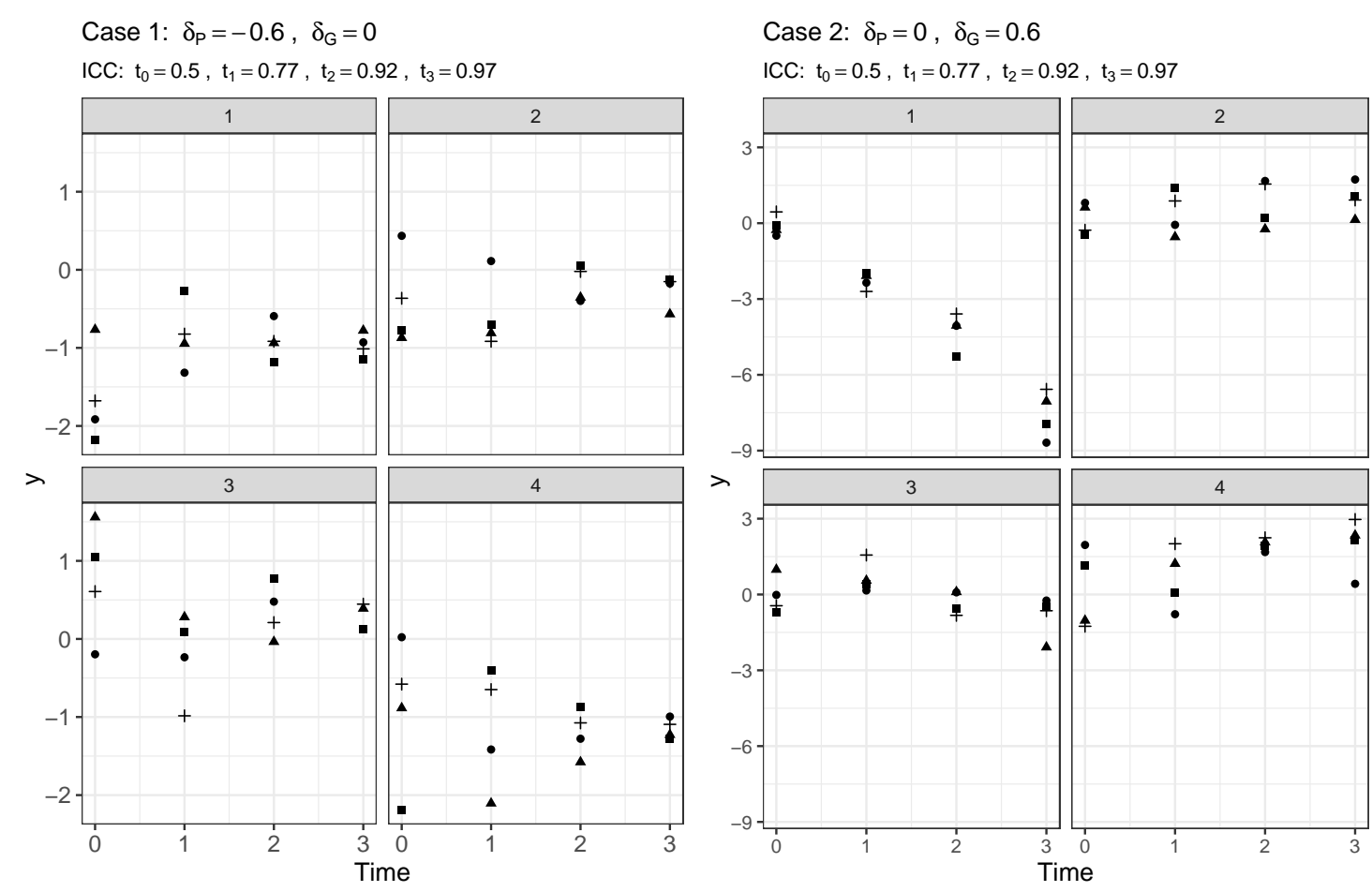

Figure 2: An illustration of two different dynamics creating the same ICC values. In the first examples (Case 1) there is consensus emergence, while in the second example (Case 2) there is an increase in group variability over time.

each measured at four time points. The first data set has a strong consensus emergence effect, with $\delta_{P}=-0.6$ (the variance between individuals is reduced by a factor of approximately 0.7 between each time point). The second data set has no consensus emergence effect but a strong effect of increased group variation, with $\delta_{G}=0.6$ (the variance between groups is increased by approximately a factor of 3.3 between each time point). The ICC values are the same for both data sets; .50, .77, .92, and .97; leading to the conclusion of a strong consensus emergence effect in both cases if used. Figure 2 shows plots of the two data sets. By visual examination, it is evident that the groups in case 1 show a pattern of consensus emergence whereas the groups in case 2 do not.

We can also show the conflation issue exactly for model (5). The ICC for model (5) is, using the exchangeability between groups and individuals within a group, at time $t$

$$
\begin{aligned}
I C C_{t} & =\operatorname{Corr}\left[P_{t i j}, P_{t k j}\right]=\frac{\operatorname{Cov}\left[P_{t i j}, P_{t k j}\right]}{\sqrt{V\left[P_{t i j}\right] V\left[P_{t k j}\right]}} \\
& =\frac{\sigma_{G}^{2} \exp \left[2 \delta_{G} t\right]}{\sigma_{G}^{2} \exp \left[2 \delta_{G} t\right]+\sigma_{P}^{2} \exp \left[2 \delta_{P} t\right]}=\frac{\sigma_{G}^{2}}{\sigma_{G}^{2}+\sigma_{P}^{2} \exp \left[2\left(\delta_{P}-\delta_{G}\right) t\right]} .
\end{aligned}
$$

As only the difference between $\delta_{P}$ and $\delta_{G}$ affects the ICC it is impossible to distinguish group level change from individual level change, and we are at risk of drawing false conclusions about consensus 
emergence.

\section{$4.2 r(t)$}

From the previous sections it is clear that ICC is not appropriate for estimating consensus emergence. We propose a new measure which is innately accounting for the aspect of time and removes the group effect that impedes ICC. Using the definition of consensus emergence in Definition 1, we argue that a natural object to study is $R_{t}=\mathbb{E}\left[\left(P_{i j}(t)-P_{k j}(t)\right)^{2}\right]$, where $j$ is a uniform draw over all groups and $i$ and $k$ are uniform draws, without replacement, among individuals in group $j$. To make the measure standardized we propose to study

$$
r(t)=\sqrt{\frac{R_{t}}{R_{0}}} .
$$

With this ratio we compare the standard deviation at time point $t$ with the standard deviation at time point 0 . By using the standard deviation rather than the variance, the unit of the statistic is the same as the data examined.

If there is no variation over time, $r(t)=1$. When the distance between group members increases over time, the ratio will be larger than one: $r(t)>1$. When the distance between group members decreases over time, i.e. there is consensus emergence, the ratio will be smaller than one: $r(t)<$ 1. Since $r(t)$ is constructed using the difference between individuals within the same group it is unaffected by a possible change in group variability and thus avoids the conflation problem of ICC.

It is often possible to derive the explicit form of the statistic for multilevel models. For instance for the model 5 specified above, the proposed ratio of interest is $r(t)=\exp \left[\delta_{P} t\right]$. In the coming section we are able to derive the explicit form of $r(t)$ for all introduced multilevel models.

It is also possible to derive a naive model free estimate of $r(t)$ but the resulting estimate will be biased (see Appendix B.2). 


\section{$5 \quad$ Advanced models for consensus emergence}

In this section we introduce multilevel models for the two types of consensus emergence defined in Section 2 heterogeneous consensus emergence and homogeneous consensus emergence. We utilize the tools illustrated in Examples 1 and 2 (Section 3) to capture different patterns of change in the error variances, and by that model the different patterns of consensus emergence.

\subsection{Consensus Emergence Model (CEM)}

The Consensus Emergence Model (CEM) (Lang et al., 2018) is a multilevel model that models linear change in latent group means. Because of the multilevel structure it allows for separation of within- and between-group variability and thus enable the study of consensus emergence in a way not possible using ICC.

The basic CEM (Lang et al., 2018, pp. 260-261) is a two-level model, with groups on level 2 and person and measurements on level 1. It is extended to a three-level model (Lang et al., 2018 pp. 264-265) by adding an intermediate level which captures prior individual experiences to account for systematic pre-study differences.

Specified with notation coherent with ours:

$$
\text { Level } 1 \text { (person - measurement) : } \begin{aligned}
P_{t i j} & =P_{i j}^{0}+G_{j}(t)+e_{t i j}^{P}, \\
e_{t i j}^{P} & \sim N\left(0, \sigma_{P}^{2} \exp \left[2 \delta_{1} t\right]\right),
\end{aligned}
$$

Level 2 (prior individual experiences) : $P_{i j}^{0}=e_{i j}^{P_{0}}$,

$$
e_{i j}^{P_{0}} \sim N\left(0, \sigma_{P_{0}}^{2}\right)
$$

Level 3 (group) : $G_{j}(t)=\mu_{0}+t \mu_{1}+e_{j}^{G_{0}}+t e_{j}^{G_{1}}$,

$$
\left[\begin{array}{c}
e_{j}^{G_{0}} \\
e_{j}^{G_{1}}
\end{array}\right] \sim N\left(\mathbf{0}, \boldsymbol{\Sigma}_{G}\right) .
$$

The model estimates a response $P_{t i j}$, on the measurement occasion $t$ for person $i$ in group $j, t=1, \ldots, t_{i}, i=1, \ldots, m_{j}, j=1, \ldots, J$. The response $P_{t i j}$ is a function of a common intercept $\mu_{0}$, a fixed-effects slope $\mu_{1}$ for time, a random group intercept $e_{j}^{G_{0}}$, a random group slope for time $e_{j}^{G_{1}}$, a random individual intercept effect $e_{i j}^{P_{0}}$, and the level 1 error term $e_{t i j}^{P}(t)$. The error variance is modeled with an exponential function of time with weight $\delta_{1}$, as illustrated in Example 1. When time is coded with 0 as the start value, and then $1,2,3$ etc, $\delta_{1}$ can be interpreted as the approximately linear change in residual variance with each measurement occasion. Positive values of $\delta_{1}$ indicates that residual variances increase over time, and negative values of $\delta_{1}$ indicates decreasing residual variances over time, i.e., consensus emergence. The placement of the exponential variance function in the CEM implies the assumption of a heterogeneous consensus emergence pattern, as level 1 errors are allowed to oscillate around the individual mean, $P_{i j}^{0}+G_{j}(t)$, between measurement occasions.

For this model it is straightforward to derive the consensus emergence statistic $r(t)$ :

$$
r(t)=\sqrt{\frac{\sigma_{P_{0}}^{2}+\sigma_{P}^{2} \exp \left[2 \delta_{1} t\right]}{\sigma_{P_{0}}^{2}+\sigma_{P}^{2}}} .
$$


The partitioning of the variances in the CEM deviates from the partitioning in the traditional three-level model (2). Usually, the first level includes (repeated) measurements, the second level individuals, and the third level groups. The three-level CEM splits the individual variance in two parts, extracting variability caused by prior individual experiences from the individual variance, making it a hybrid between the classic two-level model (1) and the three-level model (2). Thus, the first level in the CEM includes both variability from (repeated) measurements and the individual, and the second level only a part of the total individual variance. Next, we show how to extend the CEM by putting it into the more "standard" multilevel structure.

\subsection{Heterogeneous Consensus Emergence Model (HetCEM)}

The work on the CEM by Lang et al. (2018) and their note on alternative parametrizations such as the observation-level slope approach (Goldstein, 2005, 2011) leads us to the idea that we, in addition to controlling for prior individual experiences, could extend the CEM by putting it into the more "standard" multilevel structure with measurements at the first level, individuals at the second and groups at the third level. This implies that the exponential variance function of time should be moved to the second level error in order to still deal with differences between individuals. By using this structure, we introduce the possibility to separate not only the prior individual experiences from the consensus estimate, but also the measurement error which can have an impact on the findings regarding consensus emergence if not properly accounted for (Lang et al., 2018).

The model is specified as follows.

$$
\begin{aligned}
& \text { Level } 1 \text { (measurement) : } Y_{t i j}=P_{t i j}+e_{t i j}^{Y}, \\
& e_{t i j}^{Y} \sim N\left(0, \sigma_{Y}^{2}\right), \\
& \text { Level } 2 \text { (person) : } P_{t i j}=G_{j}(t)+e_{i j}^{P_{0}}+e_{t i j}^{P_{1}}, \\
& e_{i j}^{P_{0}} \sim N\left(0, \sigma_{P_{0}}^{2}\right), \\
& e_{t i j}^{P_{1}} \sim N\left(0, \sigma_{P_{1}}^{2} \exp \left[2 \delta_{1} t\right]\right), \\
& \text { Level 3 (group) : } G_{j}(t)=\mu_{0}+t \mu_{1}+e_{j}^{G_{0}}+t e_{j}^{G_{1}}, \\
& {\left[e_{j}^{G_{0}}\right.} \\
&\left.e_{j}^{G_{1}}\right] \sim N\left(\mathbf{0}, \mathbf{\Sigma}_{G}\right) .
\end{aligned}
$$

Like the CEM, the HetCEM assumes a heterogeneous consensus emergence pattern. The difference of the HetCEM compared to the CEM is that Level 2, in addition to the prior individual experiences $e_{i j}^{P_{0}}$, also includes individual errors $e_{i j}^{P_{1}}(t)$, which is where we expect a possible consensus emergence effect to show. The individual errors are allowed to oscillate around the individual mean $G_{j}(t)+e_{i j}^{P_{0}}$ and because we have the Level 1 error term, $e_{t i j}^{Y}$, capturing the measurement error, the HetCEM should give a more precise estimate of consensus emergence. The rest of the parameters in the model are the same as for the CEM.

It is possible to use $\delta_{1}$ in a similar way as in the CEM, to calculate how much the standard deviation is reduced between time points. Due to the Taylor expansion of the exponential function when $\delta_{1}$ is small it is approximately the percentage change. We do however propose to evaluate the effect size by the $r(t)$ statistic rather than using $\delta_{1}$ directly because for larger magnitudes of $\delta_{1}$, the approximation of the percentage change becomes problematic. The $\mathrm{r}(\mathrm{t})$ gives the effect size 
in terms of to what extent the variance has been reduced at a certain time point compared to the initial time point regardless the magnitude of $\delta_{1}$.

For this consensus emergence model the $r(t)$ statistic is

$$
r(t)=\sqrt{\frac{\sigma_{P_{0}}^{2}+\sigma_{P_{1}}^{2} \exp \left[2 \delta_{1} t\right]}{\sigma_{P_{0}}^{2}+\sigma_{P_{1}}^{2}}} .
$$

\subsection{Homogeneous Consensus Emergence Model (HomCEM)}

To capture heterogeneous consensus emergence, we need to include the exponential function of time in the variance of the person-level error (either on person - measurement level as in the CEM, or on person level as in the HetCEM). This ensures the possibility for each individual to give an estimate that, from time to time, oscillates above and below the group mean, with decreasing magnitude of the distance from the group mean. For homogeneous consensus emergence, all measurements from a specific individual will be either above or below the group mean, with the distance from the group mean decreasing over time. This can be modelled by moving the exponential function out of the variance of the error term $e_{i j}^{P_{1}}$, and instead multiply the error term itself with the exponential function of time with weight $\delta_{1}$, as illustrated in Example 2, We specify this model below.

$$
\text { Level } \left.1 \text { (measurement) : } \begin{array}{rl}
Y_{t i j} & =P_{i j}(t)+e_{t i j}^{Y}, \\
e_{t i j}^{Y} & \sim N\left(0, \sigma_{Y}^{2}\right), \\
\text { Level } 2 \text { (person) : } P_{i j}(t) & =G_{j}(t)+e_{i j}^{P_{0}}+\exp \left[\delta_{1} t\right] e_{i j}^{P_{1}}, \\
e_{i j}^{P_{0}} & \sim N\left(0, \sigma_{P_{0}}^{2}\right), \\
e_{i j}^{P_{1}} & \sim N\left(0, \sigma_{P_{1}}^{2}\right), \\
\text { Level 3 (group) : } G_{j}(t) & =\mu_{0}+t \mu_{1}+e_{j}^{G_{0}}+t e_{j}^{G_{1}}, \\
{\left[e_{j}^{G_{0}}\right.} \\
\left.e_{j}^{G_{1}}\right]
\end{array}\right]
$$

The parameters of this model and the interpretation of $\delta_{1}$ are the same as in the HetCEM, the only difference is the assumed pattern of consensus emergence.

For the HomCEM, although the different assumption about the pattern of consensus emergence, the $r(t)$ statistic is the same as for the HetCEM:

$$
r(t)=\sqrt{\frac{\sigma_{P_{0}}^{2}+\sigma_{P_{1}}^{2} \exp \left[2 \delta_{1} t\right]}{\sigma_{P_{0}}^{2}+\sigma_{P_{1}}^{2}}} .
$$

\section{Gaussian processes for consensus emergence}

Here we explain how to use Gaussian Processes (GPs) in consensus emergence models, which gives a more flexible model than the linear component in the mixed model that we have introduced so far. GPs have been widely used in many different fields like spatial statistics for interpolation, (Stein, 2012), Machine learning (Rasmussen, 2003), or psychology (Chen \& Zhang, 2020). The usage of GPs in multilevel modeling is not new, Diggle (1988) introduced it in a two level-model setting. 
Typically in the multilevel literature, one uses linear or some polynomial basis to describe latent individual process or group process, $P(t)$ or $G(t)$. This basis can be viewed as a low rank latent process. Here low rank refers to the fact that $P(t)$ can be defined by few deterministic functions each multiplied by a random scalar. For instance in a two level CEM the group processes is often of the form $G_{k}(t)=1 G_{j 0}+G_{j 1} t$. Thus this is a low rank processes consisting of two deterministic functions, 1 and $t$, and two random scalars, $Z_{j 0}$ and $Z_{j 1}$.

The necessity for a more "complicated" model can be questioned. Because we are often interested in the ratios of variance from different levels- like individual vs measurement or group variation- it is important to capture the relevant dependence (covariance) structure of the data. For example, Asar et al. (2020) studied the effect on fixed effects and the corresponding confidence intervals (CI) when using low rank models and GPs (and non-Gaussian processes). The conclusion was that it was crucial for the CI (for coverage) to use a model with the correct correlation structure, and that low rank models could not capture the behavior of the GPs. We will later see in the Sherif data example that capturing this behavior can give a large modeling improvement, when replacing the linear random effect with a GP for the group means. A GP is, typically, more flexible then the low rank alternatives but often without introducing too many parameters that need to be estimated. Further unlike the low rank models the GP is continuously defined. This means that irregular observations are easily handled, thereby removing the need for data imputation or missing data handling.

In the following sections we first give the basics of GPs and then introduce how to add consensus emergence to a Gaussian process.

\subsection{Overview of Gaussian processes}

For a Gaussian process, in a temporal setting, the process at time $t, G P(t)$ is a Normal random variable. For two time points, the two corresponding Normal random variables are correlated, and typically the closer the two time points are, the more dependent the variables are. A more exact definition is that for every set of time points $\mathbf{t}=\left[t_{1}, \ldots, t_{n}\right], G P(\mathbf{t})$ defines a multivariate normal random variable:

$$
G P(\mathbf{t}) \sim \mathcal{N}(\boldsymbol{\mu}(\mathbf{t}), \boldsymbol{\Sigma}(\mathbf{t}))
$$

where $\boldsymbol{\mu}(\mathbf{t})$ is a $n \times 1$ mean vector and $\boldsymbol{\Sigma}(\mathbf{t})$ is a $n \times n$ covariance matrix. Here $\boldsymbol{\Sigma}$ determines the dependence between the time point, typically there is a parametric form of the entries of the matrix. One such example is the exponential covariance function, where the covariance between two time points $G P\left(t_{1}\right)$ and $G P\left(t_{2}\right)$ is given by

$$
\Sigma\left(t_{1}, t_{2}\right)=\sigma^{2} \exp \left(-\frac{\left|t_{1}-t_{2}\right|}{\kappa}\right)
$$

where $\sigma^{2}$ is the variance of the process, and $\kappa$ is the scale of the dependence. The, perhaps, easiest way to interpret the effect of $\kappa$ is through the practical range which defines the distance, $d^{r}$, such that the correlation between two variables $G P\left(t_{1}\right)$ and $G P\left(t_{2}\right)$ at distance $d^{r}$ apart is 0.05 . The relation between $\kappa$ and $d^{r}$ is $\kappa=\frac{d^{r}}{-\log (0.05)}$ thus a higher $\kappa$ gives a higher practical range. If one has observations on a regular scale, i.e. $t_{1}=1, t_{2}=2, \ldots, \ldots t_{n}=n$, then the exponential covariance function is the same as the covariance of a regular auto regressive process of order one (AR1). The advantage with formulating a continuous process, compared to a AR process, is that it is also defined for irregular observations. 
To incorporate the GP in a multilevel setting one can in level 2 or level 3 replace the random term $e$ with GP. We illustrate this in Example 3, where we add a GP at the individual or person level.

Example 3. Consider the following model:

$$
\begin{aligned}
& \text { Level } 1 \text { (measurement): } Y_{t i j}=P_{i j}(t)+e_{t i j}^{Y}, \\
& e_{t i j}^{Y} \sim N\left(0, \sigma_{Y}^{2}\right) \\
& \text { Level 2 (person): } P_{i j}(t)=G_{j}(t)+e_{i j}^{P_{0}}+e_{i j}^{P_{1}}(t) \text {, } \\
& e_{i j}^{P_{0}} \sim N\left(0, \sigma_{P_{0}}^{2}\right), \\
& e_{i j}^{P_{1}}(t)=G P_{i j}(t) \text {, } \\
& \text { Level } 3 \text { (group): } G_{j}(t)=\mu_{0}+t \mu_{1}+e_{j}^{G_{0}}+t e_{j}^{G_{1}} \text {, } \\
& {\left[\begin{array}{c}
e_{j}^{G_{0}} \\
e_{j}^{G_{1}}
\end{array}\right] \sim N\left(\mathbf{0}, \boldsymbol{\Sigma}_{G}\right) .}
\end{aligned}
$$

Instead of using a random intercept, or error term, for each individual, as in the previous multilevel models, we let the individual term be a continuous process. In Figure 3 we illustrate four different individuals drawn from the same group $\left(G_{j}(t)=0+0.2 t\right)$. The observations are irregularly spaced with a measurement error, $\sigma_{e}$, of 0.1. Notice that $P_{i j}(t)$ is defined for all values of $t$ not only the measurement locations, as illustrated by the gray line.

\subsection{Consensus emergence}

There is no standard option to incorporate consensus emergence in a GP. We introduce a such an option. As the concept of consensus emergence indicate a change over time, the resulting process can not be a stationary process. We limit ourselves to add consensus emergence to a process with exponential covariance. However, it is easy to generalize it to any stationary GP. The idea is to add an exponential scaling to the covariance, i.e.

$$
\Sigma\left(t_{1}, t_{2}\right)=\sigma^{2} \exp \left(\delta\left(t_{1}+t_{2}\right)\right) \exp \left(-\frac{\left|t_{1}-t_{2}\right|}{\kappa}\right) .
$$

Here $\delta$ has the same effect as $\delta_{1}$ in the previous consensus emergence models. This can be seen if one calculates $r(t)$ :

$$
r(t)=\sqrt{\frac{\sigma_{P_{0}}^{2}+\sigma_{P_{1}}^{2} \exp \left[2 \delta_{1} t\right]}{\sigma_{P_{0}}^{2}+\sigma_{P_{1}}^{2}}} .
$$

Here we see the advantage with using the $r(t)$ statistic. By looking at $r(t)$ we can identify how the consensus emergence evolves over time. For example, one can see if the model assumes linear or exponential consensus emergence over time. We can not however identify which type of consensus emergence is present in data, i.e. one can not separate heterogeneous from homogeneous. 


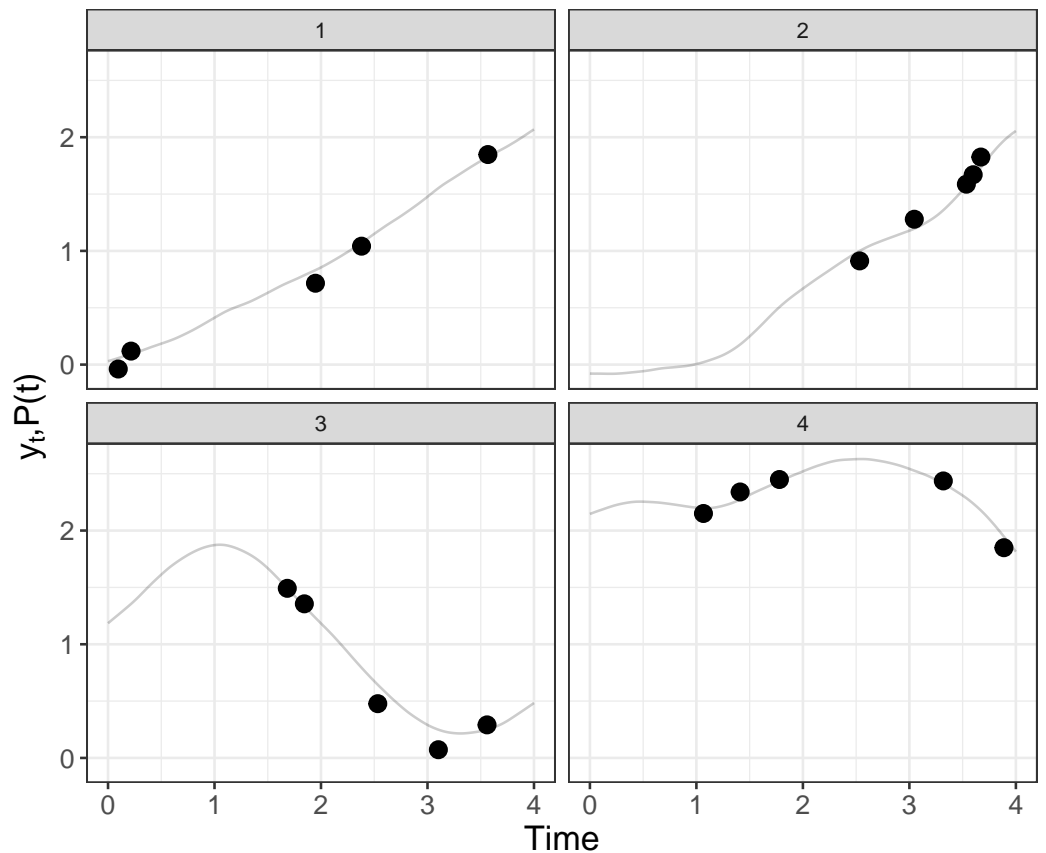

Figure 3: Observations from four individuals (dots) from a multilevel model. The lines represents the latent $P(\mathbf{t})$. The parameters are set in Example 3 . 
Thus to create a GP model with consensus emergence we add a GP with the covariance defined by (9) on Level 2 in Example 3 . We show this in Example 4.

Example 4. In Figure 4 we show the effect of adding a consensus parameter to the latent processes in Example 3. Latent processes with a consensus parameter $\delta=-0.2$ are displayed as black dashed lines, which are the same as the lines in Figure 3. One can see that in the end the solid red lines are closer to each other compared to the dashed black lines (which corresponds to the same processes without consensus).

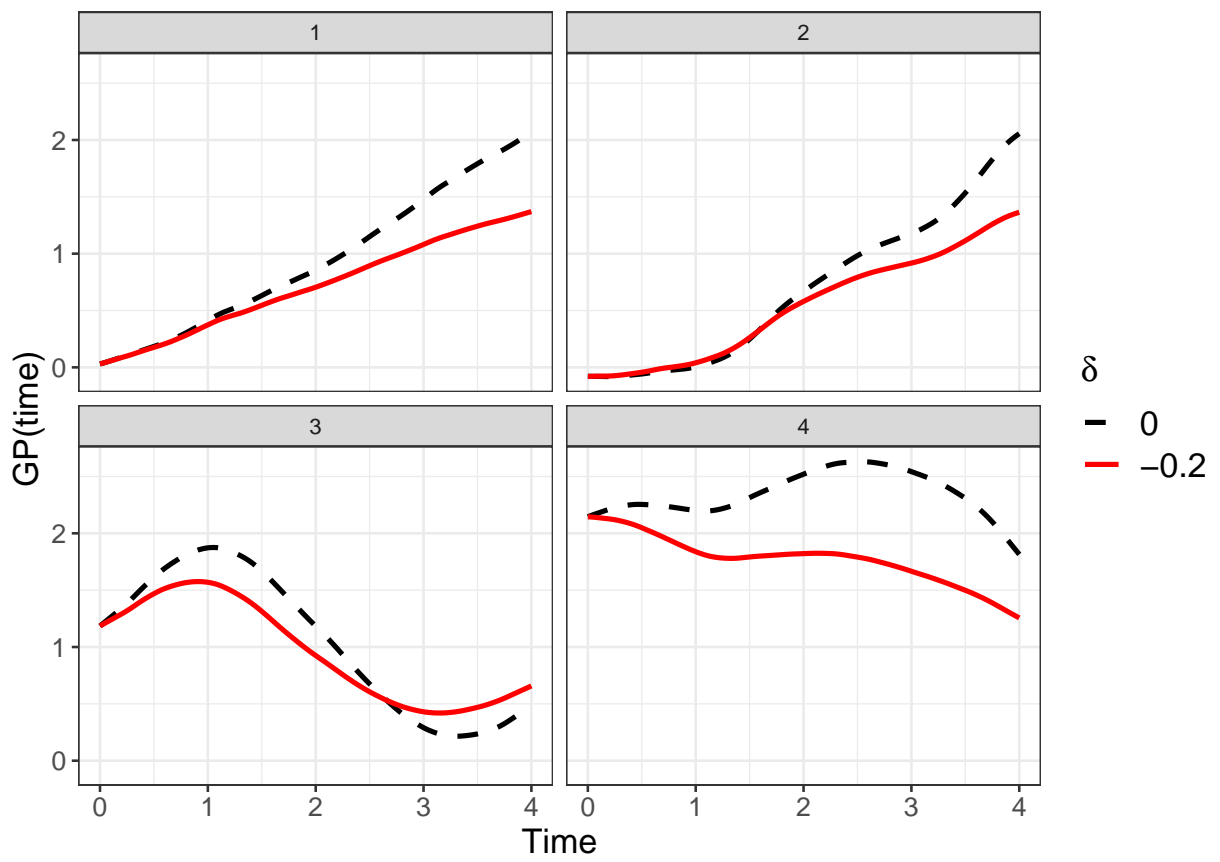

Figure 4: The black dashed lines represent the latent processes, $P_{j}(\mathbf{t})$, in Figure 3 . The solid red line is the same realisation but with an added consensus parameter $\delta=-0.2$. 


\section{Estimation on data and a simulation}

\subsection{Sherif autokinetic effect data}

Sherif's (1935) study of the autokinetic effect is an early study of consensus emergence. The autokinetic effect occurs when a person is repeatedly presented with a small light in a completely dark room, so that the person has no basis of comparison to where the light is presented. Then the light will seem to move even if it is not. Sherif divided 24 individuals into eight groups, and asked them to estimate the distance the light moved. There were three sessions for each group, and one individual session for each participant. The members of four of the groups had their individual sessions before the group sessions and the members of the other four groups had their individual sessions after the group sessions. At each session, the light was presented 100 times. The data published in Sherif (1935) consist of the median values for each subject and session (Tables XVII-XXIV, pp. 56-60). The data are available in the MMP package.

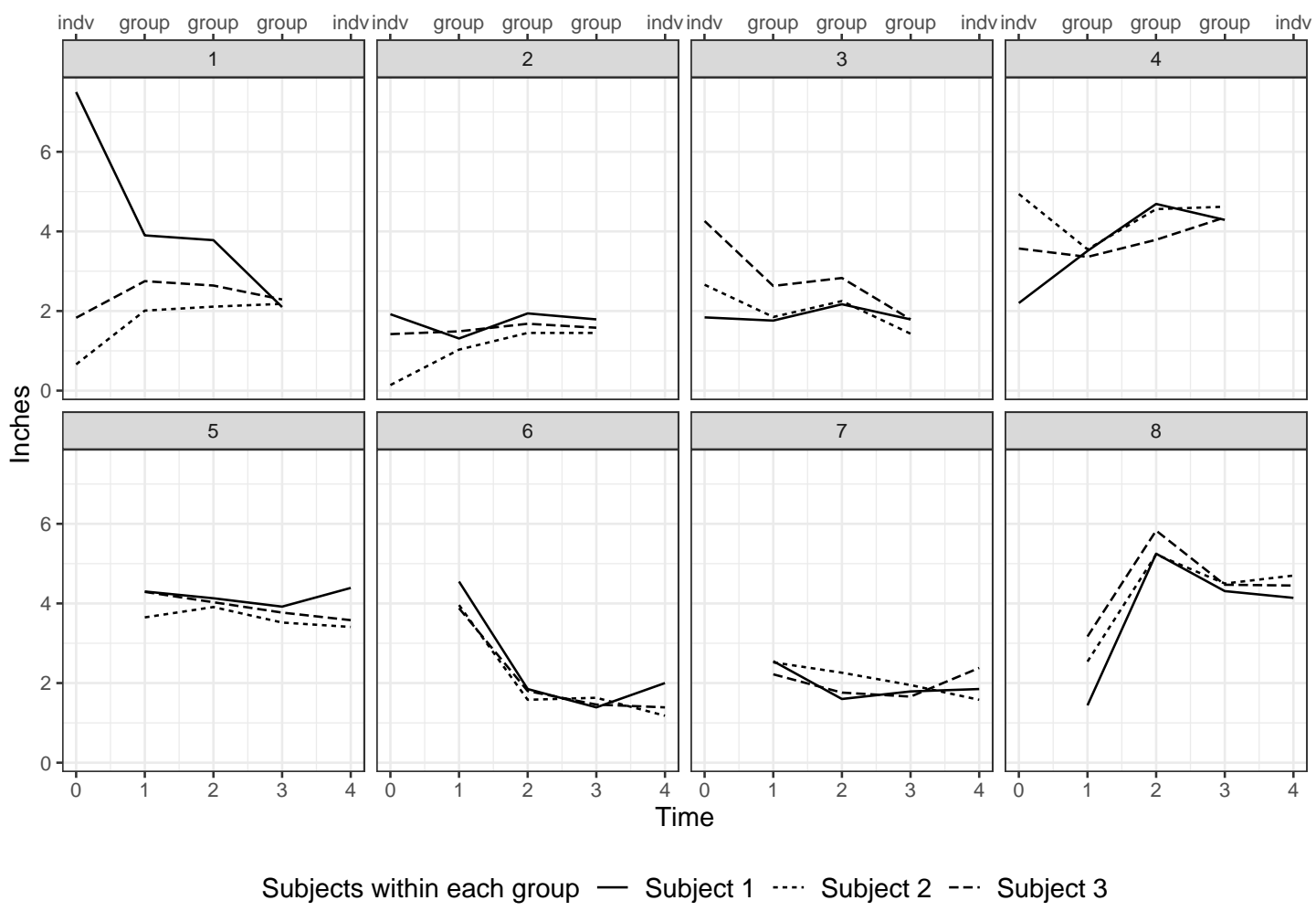

Figure 5: Data from Sherif (1935)

We repeat the analysis of the Sherif data from Lang and Bliese (2019) but we also include the first time point where some individuals gave distance estimates at an individual session before the group sessions. The inclusion of the first estimate allows for a stronger estimation of the consensus emergence. That is, we have a time point with only individual variability and no group effect. The 
Sherif data are ideal to illustrate the conflation problem discussed in previous sections due to the strong effects, both in terms of consensus emergence and change the group means over time.

Examining the data (Figure 5) the pattern of consensus emergence is not obvious. Groups 1 and 8 show signs of homogeneous consensus emergence. For the other groups, there are some overlap of the individual lines. This indicates that the pattern may be more of a heterogeneous character. The effect of group mean change over time can be confounded with the effect consensus emergence, if this first effect is not properly accounted for.

We work in two stages. First, we fit the consensus models using linear trends to describe the group dynamics. Examining the data, the linear assumption might be violated. Second and consequently, we fit the consensus models again but with a more dynamic structure for the group level change. We will show that the more complex team dynamic model fit the data better. ${ }^{1}$

\section{Linear team dynamics}

We fit the heterogeneous consensus emergence model (HetCEM), the homogeneous consensus emergence model (HomCEM) and the Gaussian processes consensus emergence model (GP CEM) defined in equations $7,8,9$ to the data.

\section{Nonlinear team dynamics}

The previous models assume that the change in the latent group means is linear over time. However, a visual examination indicates that the group mean may change more between the first two time points and less between the later time points. That is, the change in latent group mean may be more adequately captured by a nonlinear trend. We can model this nonlinear process where the magnitude of change decrease over time using a Gaussian process with the same covariance structure as for the GP with consensus emergence defined in section 6.2. Thus we replace the linear group level

$$
G_{j}(t)=\mu_{0}+t \mu_{1}+e_{j}^{G_{0}}+t e_{j}^{G_{1}}
$$

with

$$
G_{j}(t)=\mu_{0}+t \mu_{1}+G P_{j}(t)
$$

where the covariance of $G P_{j}$ is given by (9). We denote the $\delta$ parameter in $(9)$ with $\delta_{G}$ and it controls how fast the group mean converges to a fixed point.

\section{Results}

Model comparisons are shown in Table 1; we present both $A I C, B I C$, the log likelihood values and finally the Akaike weight (Burnham \& Anderson, 2002). The Akaike weights can informally be thought of as how likely each model is to be best according to the $A I C$. Here we can see that, out of the linear models, the HetCEM appears to fit the data best according to both $A I C$ and BIC, but it is clear that the nonlinear team models fit the data better. This suggests that the linear group models indeed fail to adequately capture the latent group mean trend. The HomCEM with a team GP is the best fitted model.

Importantly, there are two major dynamics of the data that changes over time. The first dynamic is the convergence of individuals, within a groups, towards the group mean (consensus emergence). The second dynamic is the stabilization of the group mean over time (not towards a joint mean).

\footnotetext{
${ }^{1}$ Code for the analysis can be found on GitHub: github.com/JonasWallin/MMP
} 
This is can, for instance, be seen in that that the group means at time point 2 is closer to the group mean at time point 3 than time point 1, i.e. the variance of $G_{i 3}-G_{i 2}$ is smaller than the variance of $G_{i 2}-G_{i 1}$.

These two separate dynamics are described by two statistics in the models introduced earlier. The consensus emergence is controlled by $\delta_{1}$, and the stabilization of group mean is controlled by $\delta_{G}$. In Table 2 we can see the estimated values of the parameter for each model. The estimated effect of both dynamics is very big, hence it is clear that we have both dynamics present in the data.

Table 1: Model comparisons for Sherif data.

\begin{tabular}{lrrrr}
\hline Model & AIC & BIC & loglik & Akaike weight \\
\hline linear team models & & & & \\
null model & 108.19 & 127.64 & -46.10 & 0.00 \\
HetCEM & 54.73 & 76.61 & -18.37 & 0.00 \\
HomCEM & 67.94 & 89.82 & -24.97 & 0.00 \\
GP & 56.73 & 81.04 & -18.37 & 0.00 \\
nonlinear team models & & & & \\
HetCEM & 41.94 & 66.25 & -10.97 & 0.00 \\
HomCEM & 25.43 & 49.75 & -2.72 & 0.65 \\
GP & 26.69 & 53.43 & -2.35 & 0.35 \\
\hline
\end{tabular}

Table 2: Parameter estimates Sherif data

\begin{tabular}{l|rrrrrrr}
\hline & \multicolumn{3}{|c}{ linear team models } & \multicolumn{3}{c}{ nonlinear team models } \\
\hline Parameters & null model & HetCEM & HomCEM & GP & HetCEM & HomCEM & GP \\
\hline Intercept & 3.05 & 3.37 & 3.09 & 3.37 & 3.32 & 3.31 & 3.32 \\
time & -0.10 & -0.23 & -0.11 & -0.23 & -0.21 & -0.20 & -0.21 \\
$\hat{\sigma}_{P_{0}}^{2}$ & 0.18 & 0.00 & 0.00 & 0.00 & 0.01 & 0.01 & 0.00 \\
$\hat{\sigma}_{P_{1}}^{2}$ & 0.00 & 5.86 & 3.26 & 5.86 & 3.07 & 3.38 & 3.20 \\
group intercept variance & 0.85 & 0.65 & 0.73 & 0.65 & 1.38 & 1.33 & 1.37 \\
group slope variance for time & 0.27 & 0.05 & 0.27 & 0.05 & & & \\
group cov(intercept, slope) & -0.27 & 0.07 & -0.22 & 0.07 & & & \\
$\hat{\sigma}_{Y}^{2}$ & 0.65 & 0.00 & 0.26 & 0.00 & 0.01 & 0.06 & 0.05 \\
$\delta_{1}$ & & -0.83 & -1.17 & -0.82 & -0.88 & -1.22 & -1.08 \\
$\delta_{G}$ (team effect) & & & & & -0.70 & -0.67 & -0.69 \\
\hline
\end{tabular}



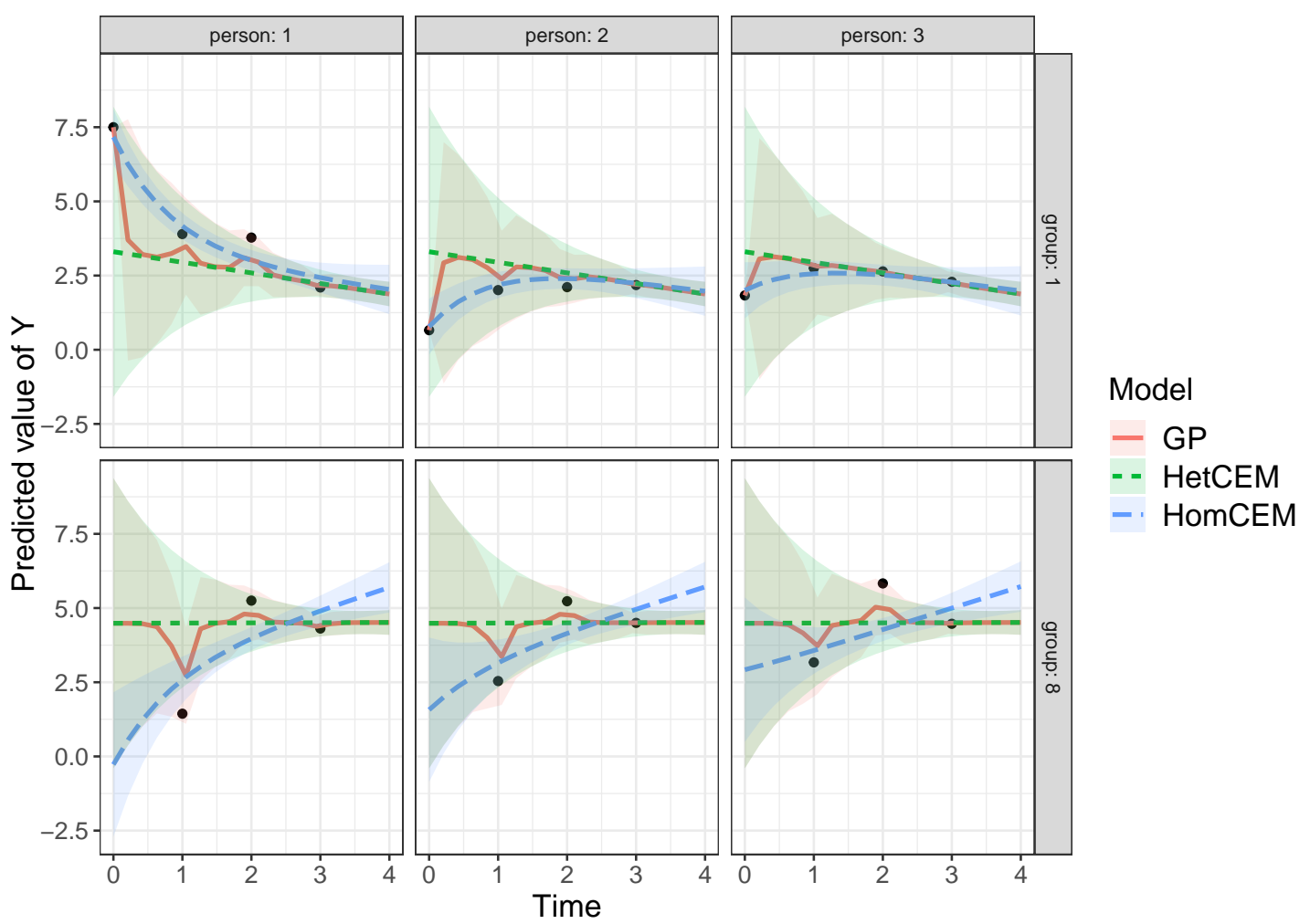

Figure 6: Smoothing distribution of where a new observation could land for the different "regular" models, for groups 1 (upper row) and 8 (lower row). Black points are the observed values. 

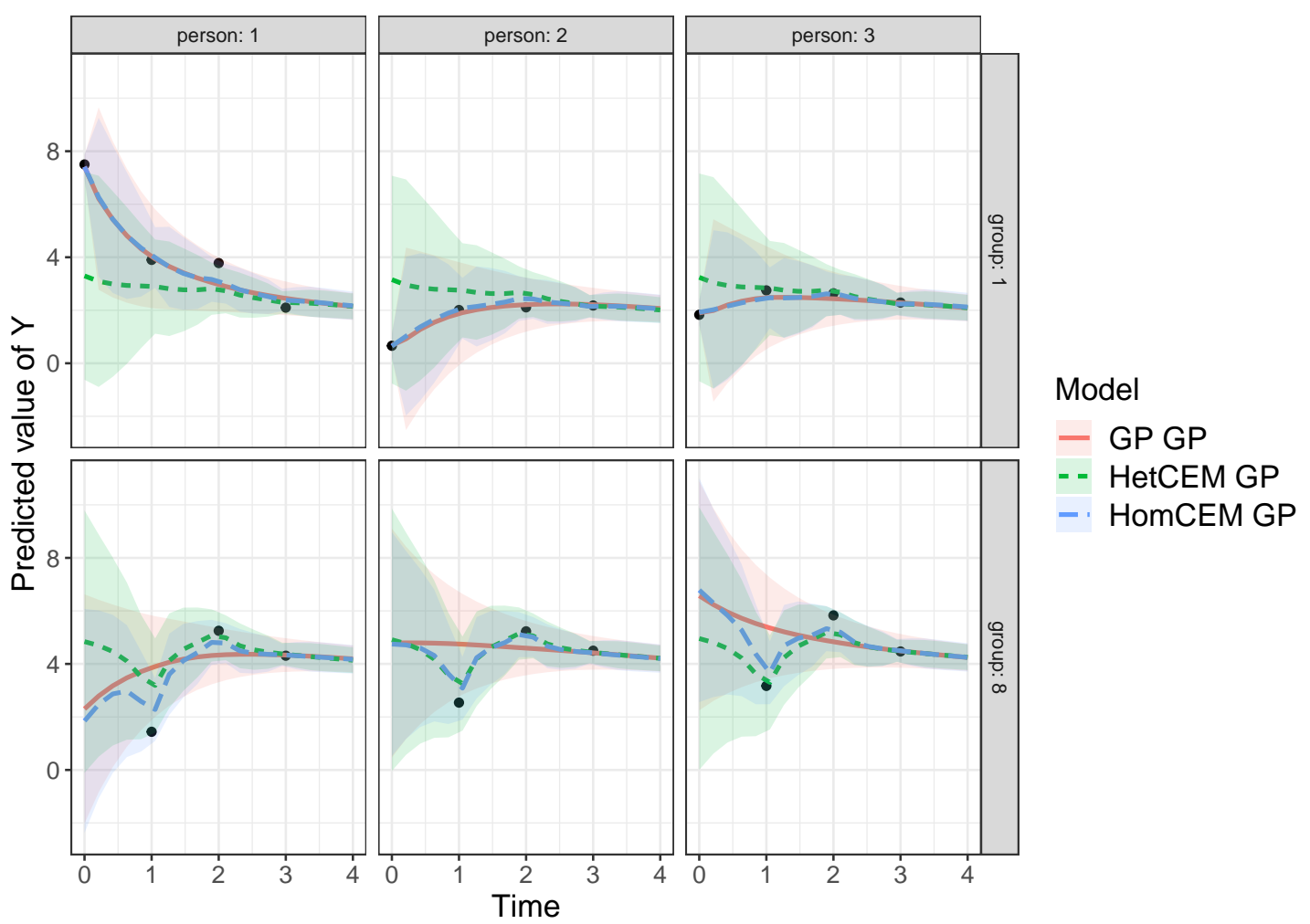

Figure 7: Smoothing distribution of where a new observation could land for the different GP teams models, for groups 1 (upper row) and 8 (lower row). Black points are the observed values.

In Figure 6 and Figure 7 , to get a further feel for the different models we plot the mean and the 95\% prediction interval for $Y(t)$ given all observations for two groups. The interpretation of the interval is that if we would make a new observation we would expect that, with $95 \%$ confidence, the observation is in the interval. Overall the patterns of the models are similar with a large variability early and decreasing towards the end. However, the variability in the nonlinear model is not due to individual variability but rather group mean variability. If we examine the observations for the individuals in group 8 we see that they vary clearly over time but not over individuals; for each time point all the observations for the group members are at basically the same location. It is also easy to see that no simple linear trend capture this behavior of the group mean over time, yet it is fundamental for describing the team dynamics. This is why the nonlinear time team models outperform the linear team models. 


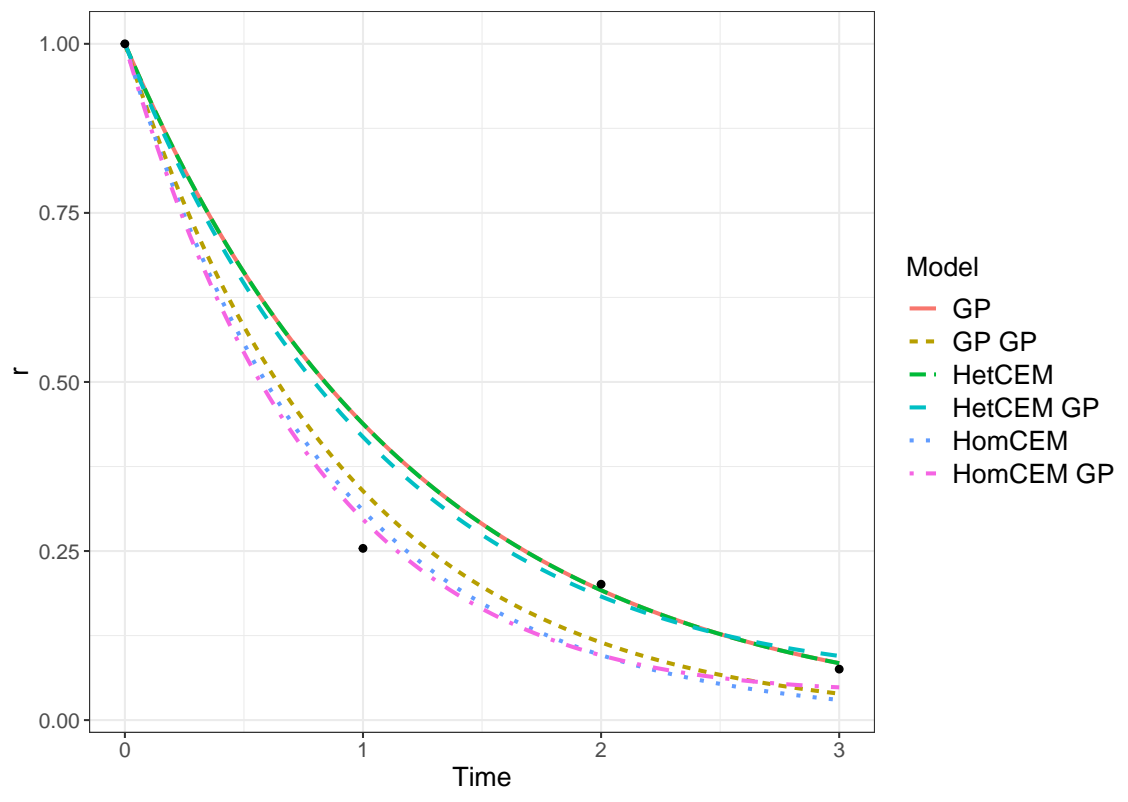

Figure 8: $r(t)$ curves for the fitted models. Black points are the empirically estimated values $\hat{r}^{y}(t)$. HomCEM models with and without team GPs find stronger consensus emergence than the other models.

Finally, regarding the consensus emergence effects for the models we can use $r(t)$ which is presented in Figure 8 for all models. The black points are the empirically, biased, estimated values $\hat{r}^{y}(t)$. All models are to a large extent able to capture the same amount of consensus emergence, despite different assumptions about the pattern of emergence. However, a remaining problem could be that the linear models (which assume no group mean convergence) are conflating the group mean convergence with the consensus emergence. When we introduce a group mean convergence parameter $\left(\delta_{G}\right.$, in Table 2 $)$, we find a strong effect for group mean convergence in the same direction as the consensus emergence ( $\delta_{1}$ in Table 2). Hence, we can't know if the linear models capture only the consensus emergence or both (the consensus emergence and the group mean convergence).

In short, it is clear from Table 1 that the models with nonlinear team dynamics fits the data much better than the models with linear team dynamics. The use of the nonlinear team models change which type of consensus emergence pattern fits the data best (from heterogeneous to homogeneous). However, it is not clear that the addition of the nonlinear team dynamics improves the estimation of the consensus emergence effect as they are similar for all models (linear and nonlinear). 


\subsection{Simulation}

As mentioned previously, the Sherif data are ideal to illustrate conflation problems due to the strong effects. As Lang, Bliese, and Runge (2019) note, extreme effects sizes like these are unlikely to be observed in most field applications. To illustrate how the HetCEM, HomCEM and GP model performs for more moderate effect sizes and different types of consensus emergence, we perform a simulation study.

For the simulations we generate four different types of data $2^{2}$

- Heterogeneous consensus emergence: where the data are generated from the HetCEM.

- Homogeneous consensus emergence: where the data are generated from the HomCEM.

- Heterogeneous consensus emergence with nonlinear group mean change: where the data are generated from the HetCEM with Gaussian processes for group means. H

- Homogeneous consensus emergence with nonlinear group mean change: where the data are generated from the HomCEM with Gaussian processes for group means.

Values used in the simulations are given in Table 3. To complement the Sherif data, which show strong effects over a short time span, we use 20 groups, each with 7 individuals measured at 7 time points.

The consensus emergence parameter $\delta_{1}$ is set to $0,-0.03,-0.05$, and -0.08 . The effects of these parameters are in line with what is argued to be reasonable effect sizes (Lang \& Bliese, 2019; Lang et al., 2018; Lang, Bliese, \& Runge, 2019).

For the simulations with nonlinear group mean change, $\sigma_{G_{0}}^{2}$ is set to be small initially to correspond to groups where a distinct climate have not yet been developed (Lang, Bliese, \& Adler, 2019). We set $\delta_{G}=0.06$ to correspond to an increase in between-group variance, arriving at $\sigma_{G_{0}}^{2}=0.1$ at time point 7 . This extra effect is added so that it should be harder to estimate the consensus emergence since the group variability is increasing, see the examples in section 4.

\section{Results}

Results of the simulations are shown in Figure 9. The overall message is that the models with homogeneous consensus emergence is better at capturing consensus emergence than models with heterogeneous consensus emergence. If there is no non linear team dynamics the homogeneous models capture the consensus emergence regardless of which type of emergence the data is generated from. However when data is generated with non linear team dynamics (with destabilization) one needs to correctly specify the consensus emergence in order to capture it.

This behavior deviates from the Sherif example where all models manage to find consensus emergence to some extent even though evidence points to a true homogeneous consensus emergence pattern. An additional simulation confirms that this behavior disappears when changing the relatively weak effects of $r\left(t_{\max }\right)$ values ranging from $0.83-0.92$ used here, to a value of 0.1 corresponding to the $r\left(t_{\max }\right)$ estimated from the Sherif data (Appendix D). For stronger consensus emergence effects, all models in the simulation find consensus emergence, although the HetCEM and GP models (with and without nonlinear team effects) still underestimates its effects. That the GP models are not able to detect consensus emergence in all the cases is strange since the GP

\footnotetext{
${ }^{2}$ Code for the simulation can be found on GitHub: github.com/JonasWallin/MMP
} 
Table 3: Parameters

\begin{tabular}{lcc}
\hline Parameter & regular models & team GP models \\
\hline$\mu_{0}$ & 3.00 & 3.00 \\
$\mu_{1}$ & 0.01 & 0.01 \\
$\sigma_{Y}^{2}$ & 0.10 & 0.10 \\
$\sigma_{P_{0}}^{2}$ & 0.10 & 0.10 \\
$\sigma_{P_{1}}^{2}$ & 0.10 & 0.10 \\
$\sigma_{G_{0}}^{2}$ & 0.05 & - \\
$\sigma_{G_{1}}^{2}$ & 0.005 & - \\
$\sigma_{G_{0} G_{1}}$ & -0.005 & - \\
$\sigma_{G P}^{2}$ & - & 0.05 \\
corr & - & 0.05 \\
$\delta_{1}$ & $0,-0.03,-0.05,-0.08$ & $0,-0.03,-0.05,-0.08$ \\
$\delta_{G}$ & - & 0.06 \\
& & \\
Number of groups, $K$ & 20 & 20 \\
Individuals per group, $M$ & 7 & 7 \\
Observations per individual, $N$ & 7 & 7 \\
Total observations & 980 & 980 \\
\hline
\end{tabular}

model can be transformed to either a Homogeneous or a Heterogeneous model. An explanation could be that one is not able to find the GP model that fits the data optimally, i.e., the numerical optimizer have not found the best parameters for the data. 


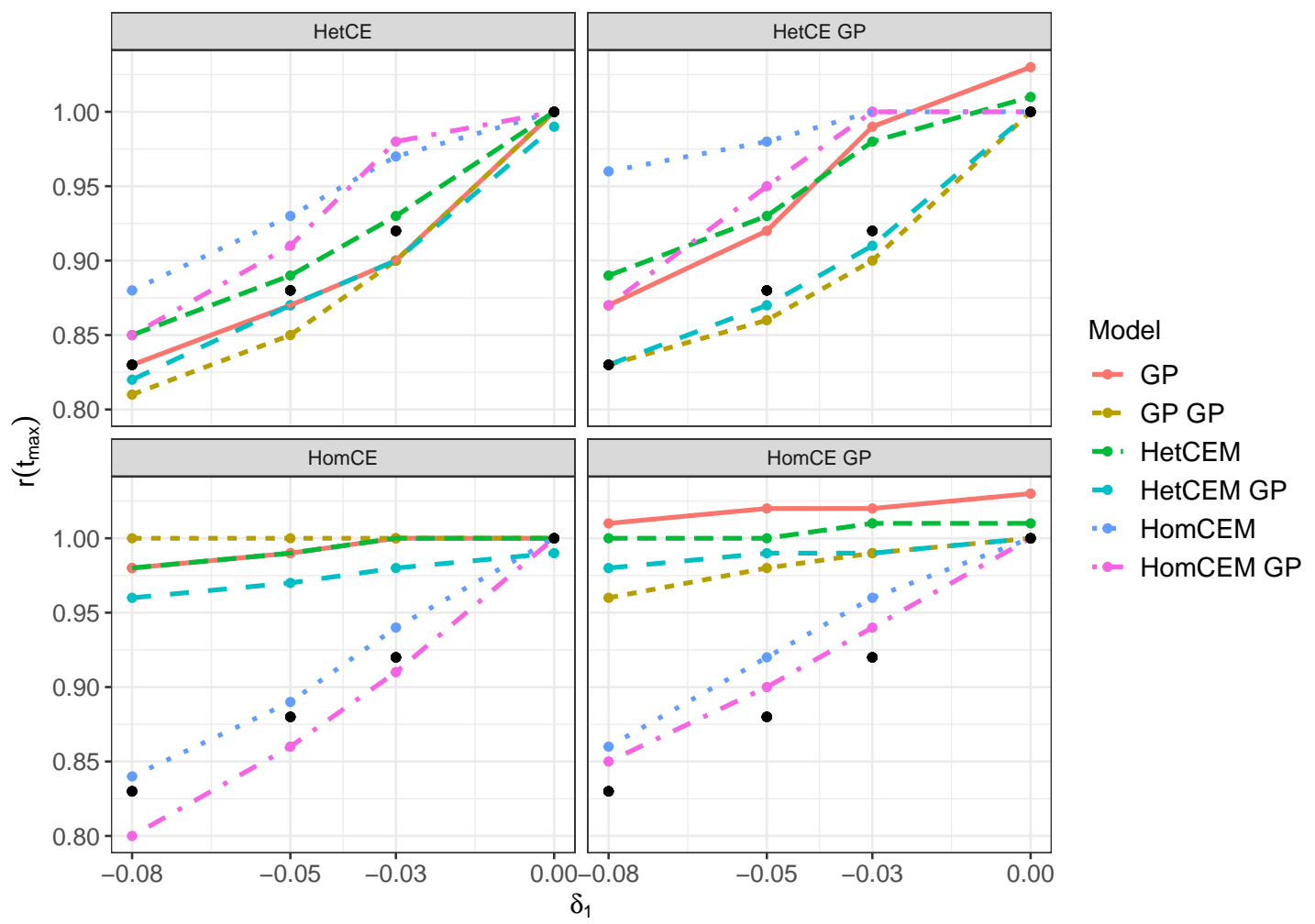

Figure 9: Median $r\left(t_{\max }\right)$ values from simulations of the four different settings: heterogeneous consensus emergence with and without a team GP effect, and homogeneous consensus emergence with and without a team GP effect. Black points are the true $r\left(t_{\max }\right)$ values.

To sum up, the simulations show the following. For moderate effect sizes, it is even more important to adapt the model to the pattern of consensus emergence and nonlinear changes in latent group means. The simulations also show that the conflation problem which ICC suffers from is not unique to ICC or completely solved by using the $r(t)$ statistic. We need to accurately capture the group level behavior in order to capture the consensus emergence. 


\section{Discussion}

Emergence describes a multilevel process where lower-level units change over time to form characteristics of higher-level units (Baronchelli, 2018, Fulmer \& Ostroff, 2016, Kozlowski et al., 2013 Wee \& Taylor, 2018). The concept of emergence and its measurement play critical roles in theory development of organizations and groups and empirical research. We described a new statistical approach to allow organizational and behavioral scholars to examine such frequent bottom-up processes over time within groups.

We provide an important extension to the concept of emergence and its measurement. We thereby contribute to recent and important developments made by Lang and colleagues on the topic. The multilevel models we developed for consensus emergence can also be used for modeling group mean change over time. We develop the model by moving the consensus emergence framework to the third level, i.e., the group level. We did this for the Sherif data set by adding Gaussian processes with decreasing variability over time. These changes both improved the fit of the model and also changed the results. We show that homogeneous not heterogeneous consensus emergence is most likely, which illustrates the importance of including group mean changes in the analysis.

\section{Methodological and theoretical implications}

First, emergence is often the theoretical basis for aggregating responses to higher-levels and doing research using higher-level constructs. That is, the evidence that emergent processes take place for specific constructs represents a critical piece of the multilevel construct validation process to support aggregation. The study of consensus emergence is important to the development of theory. Similar to other areas of research, studies of emergence need to have acceptable models and statistics to determine the existence and nature of our temporal patterns to help to advance knowledge. Based on recent advancement in the field, we have developed new more versatile models and statistics to detect and assess different types of emergence. Using real and simulated data, we show how our models are improving and extending the statistical analysis of consensus emergence. In the simulation study we observe that in some cases, if one incorrectly specified the type of consensus emergence, then one was unable to detect any consensus. This observation goes against the result found when examining the Sherif data. This discrepancy is probably due to the magnitude of the consensus emergence which was extreme in the Sherif data (reach almost complete consensus) whereas a much weaker effect was used in the simulation study.

We show specifically that our models are more effective than previous when there are weak effects of emergence in data. Specifically, we define two types of consensus emergence models, which we denote Homogeneous and Heterogeneous Consensus Emergence, HomCEM and HetCEM. In addition, we provide R-based tools to more easily implement our models.

Second, because we identify different patterns of consensus emergence, we provide a common mathematical definition of what consensus emergence is in multilevel models. We use this definition to construct a statistic, $\mathrm{r}(\mathrm{t})$, to measure the strength of consensus emergence.

Third, to manage the, sometimes, complex dynamics existing in emergent processes, we show how to use Gaussian processes (GPs) for multilevel consensus emergence. GPs are applied to capture complex dynamics in many different areas like spatial statistics (Matérn, 2013), machine learning (Rasmussen, 2003) and inverse problems (Stuart, 2010). GPs are more flexible than standard random effect in multilevel models and can capture nonlinear time dynamics. Further, the GP are continuously defined. This means that irregular observations are easily handled, thereby 
removing the need for data imputation or missing data management. This is potentially an important advantage in applied research where missing data can be prevalent. Overall, we are proposing a flexible approach to detect consensus emergence, and we hope that the models and statistics presented can open up new avenues for empirical research on emergence.

\section{Practical implications}

A practical implication is that research using our tool may provide useful new temporal insights for organizations. Second, while our paper is on inference, the models introduced can be used to predict future outcomes. This provides a way to develop and test procedures to predict emergence. Again, using the example of organizational climate, researchers and practitioners could asses if a specific training program causes organizational members to more rapidly develop shared organizational climates. Such a study would be to investigate mean change over time, but in addition to mean change, it would be important to examine patterns of emergence among members to enhance consensus.

\section{Limitations and Future directions}

We identify several weaknesses in our current work and opportunities for future research. First, we assume that the groups are independent from each other. This is not necessarily the case in all empirical settings, as we might consider field studies where groups are dependent of each other, such as working within the same corporate setting or in multi-team systems (Luciano et al., 2020, Zaccaro et al., 2020). This would mean that we would need to model dependency also at the group level.

Second, in applied research the data are rarely neat univariate normal random variables, but rather often multivariate (e.g., multiple constructs in a survey) and non-Gaussian (e.g., binary or count data). Thus an important future direction is to develop a methodology for multivariate observations and putting the models in a generalized linear models (GLM) setting.

Third, group and team dynamics are likely to be anything but linear, something most multilevel models have assumed so far(Kozlowski \& Chao, 2018, Ramos-Villagrasa et al., 2018, Wang et al., 2016 Wee \& Taylor, 2018). We have tried to develop a GP model that better fit such dynamic data. We have seen here that in emergence research the pattern for group mean convergence is an important dimension to more fully account for consensus. The adaptation of GP and similar dynamic models will continue to enrich our understanding of group and team dynamics. The use of longitudinal data collected with a design taking temporal changes into account are paramount. The analysis of such data will be enriched with dynamic models that allow for better fit of such data than more traditional linear ones. We encourage research to continue develop our thinking here as this is a first attempt with some documented weaknesses. For example, while we assess better fit, the coefficients are not always improved when using GP. Future research will need to continue to improve the solutions here.

Fourth, we have assumed equal importance among our team members. However, not all team members are always equal (DeRue et al., 2010). Dispersion in teams can exist for different reasons and, independent of its magnitude, may meaningfully impact consensus emergence at the team level. For example, team members might hold different positions or different roles within the same group may differ in how they experience the team. Team leadership is such an example. Team leadership is an important topic in applied research and in consensus emergence. The team leader can have an important impact on how emergence is shaped by imposing their values and ideas on 
other team members by authority or conviction (Cook et al., 2020; Hanna et al., 2021; Solinger et al., 2020). The importance of leadership in an emergent process might be a complicated problem to solve unless the leader is already defined. If on the contrary, the leader is not yet defined but is emerging, that is, a member of the team is increasingly having a relatively strong weight in the team, we have not even considered this possibility in our current models of emergence. If we do not know ex ante who the dominating individual is and we suspect that emergence of leadership will happen, then we have to develop models that takes this uncertainty into account.

Finally, we have not addressed at all missing data. Missing data are often prevalent in longitudinal data. We have already suggested that GPs are more flexible and continuously defined. Irregular observations are easily handled, potentially removing the need for data imputation or missing data management. For missing completely at random, Gaussian processes are ideal. However they have limited value for missing at random or missing not at random. To incorporate GPs and missing values into the multilevel consensus emergence is an important extension.

\section{Conclusion}

Bottom-up emergent processes are theoretically important to understand social dynamics in groups and organizations. We have here built on Lang et al's CEM to study emergence of shared constructs. Specifically, we expand the consensus emergence concept and introduce two models to distinguish between different patterns of consensus emergence, homogeneous and heterogeneous. We also introduce a model-invariant statistic, $\mathrm{r}(\mathrm{t})$ to measure the strength of the consensus; and to compare between different models and patterns of consensus emergence. To capture complex dynamics in emergent processes such as nonlinear changes in group means over time, we extend these models with Gaussian Processes. We show that the conclusions on the pattern of consensus change when adequately modelling the nonlinear group mean change over time. We believe the methodology can open further avenues in the study of consensus emergence and related bottom-up processes in organizations.

\section{Acknowledgment}

This work has been supported by the Swedish Research Council under grant No. 2018-01726. 


\section{References}

Allen, N. J., \& O’Neill, T. A. (2015). The trajectory of emergence of shared group-level constructs. Small Group Research, 46(3), 352-390. https://doi.org/10.1177/1046496415584973

Asar, Ö., Bolin, D., Diggle, P. J., \& Wallin, J. (2020). Linear mixed effects models for non-gaussian continuous repeated measurement data. Journal of the Royal Statistical Society: Series $C$ (Applied Statistics).

Baronchelli, A. (2018). The emergence of consensus: A primer. Royal Society Open Science, 5(2), 172189.

Bartko, J. J. (1966). The intraclass correlation coefficient as a measure of reliability. Psychological Reports, 19(1), 3-11. https://doi.org/10.2466/pr0.1966.19.1.3

Bedau, M. A. (1997). Weak emergence. Philosophical perspectives, 11, 375-399.

Bliese, P. D. (2000). Within-group agreement, non-independence, and reliability: Implications for data aggregation and analysis. In Multilevel theory, research and methods in organizations: Foundations, extensions and new directions (pp. 349-381). Jossey-Bass.

Bliese, P. D., \& Ployhart, R. E. (2002). Growth modeling using random coefficient models: Model building, testing, and illustrations. Organizational Research Methods, 5(4), 362-387.

Burnham, K. P., \& Anderson, D. R. (2002). Model selection and multimodel inference : A practical information-theoretic approach. Springer.

Chen, Y., \& Zhang, S. (2020). A latent gaussian process model for analysing intensive longitudinal data. British Journal of Mathematical and Statistical Psychology, 73(2), 237-260.

Cook, A. S., Zill, A., \& Meyer, B. (2020). Observing leadership as behavior in teams and herdsan ethological approach to shared leadership research. The Leadership Quarterly, 31(2), 101296.

Cronin, M. A., Weingart, L. R., \& Todorova, G. (2011). Dynamics in groups: Are we there yet? Academy of Management Annals, 5(1), 571-612.

DeRue, D. S., Hollenbeck, J., Ilgen, D., \& Feltz, D. (2010). Efficacy dispersion in teams: Moving beyond agreement and aggregation. Personnel Psychology, 63(1), 1-40. https://doi.org/ https://doi.org/10.1111/j.1744-6570.2009.01161.x

Diggle, P. J. (1988). An approach to the analysis of repeated measurements. Biometrics, 959-971.

Fisher, R. A. (1932). Statistical methods for research workers. (6th ed.). Edinburgh, Oliver \& Boyd.

Fulmer, C. A., \& Ostroff, C. (2016). Convergence and emergence in organizations: An integrative framework and review. Journal of Organizational Behavior, 37, S122-S145.

Goldstein, H. (2005). Heteroscedasticity and complex variation. Encyclopedia of statistics in behavioral science, 2, 790-795.

Goldstein, H. (2011). Multilevel statistical models. John Wiley \& Sons.

Hanna, A. A., Smith, T. A., Kirkman, B. L., \& Griffin, R. W. (2021). The emergence of emergent leadership: A comprehensive framework and directions for future research. Journal of Management, 47(1), 76-104.

Humphrey, S. E., \& Aime, F. (2014). Team microdynamics: Toward an organizing approach to teamwork. Academy of Management Annals, 8(1), 443-503.

Humphrey, S. E., \& LeBreton, J. M. (2019). The handbook of multilevel theory, measurement, and analysis. American Psychological Association.

Kauffman, S., \& Clayton, P. (2006). On emergence, agency, and organization. Biology and Philosophy, 21 (4), 501-521. 
Kozlowski, S. W., \& Chao, G. T. (2018). Unpacking team process dynamics and emergent phenomena: Challenges, conceptual advances, and innovative methods. American Psychologist, $73(4), 576$.

Kozlowski, S. W., Chao, G. T., Grand, J. A., Braun, M. T., \& Kuljanin, G. (2013). Advancing multilevel research design: Capturing the dynamics of emergence. Organizational research methods, 16(4), 581-615.

Lang, J. W. B., \& Bliese, P. D. (2019). A temporal perspective on emergence: Using three-level mixed-effects models to track consensus emergence in groups. In The handbook of multilevel theory, measurement, and analysis (pp. 519-540). American Psychological Association.

Lang, J. W. B., Bliese, P. D., \& Adler, A. B. (2019). Opening the black box: A multilevel framework for studying group processes. Advances in Methods and Practices in Psychological Science, 2(3), 271-287.

Lang, J. W. B., Bliese, P. D., \& de Voogt, A. (2018). Modeling consensus emergence in groups using longitudinal multilevel methods. Personnel Psychology, 71(2), 255-281.

Lang, J. W. B., Bliese, P. D., \& Runge, J. M. (2019). Detecting consensus emergence in organizational multilevel data: Power simulations. Organizational Research Methods, 1-23.

Larson, N. L., McLarnon, M. J., \& O'Neill, T. A. (2020). Challenging the "static" quo: Trajectories of engagement in team processes toward a deadline. Journal of Applied Psychology, 105 (10), 1145 .

Lester, H. F., Cullen-Lester, K. L., \& Walters, R. W. (2021). From nuisance to novel research questions: Using multilevel models to predict heterogeneous variances. Organizational Research Methods, 24(2), 342-388.

Little, T. D. (2013). Longitudinal structural equation modeling. Guilford press.

Luciano, M. M., Nahrgang, J. D., \& Shropshire, C. (2020). Strategic leadership systems: Viewing top management teams and boards of directors from a multiteam systems perspective. Academy of Management Review, 45(3), 675-701.

Marks, M. A., Mathieu, J. E., \& Zaccaro, S. J. (2001). A temporally based framework and taxonomy of team processes. Academy of management review, 26(3), 356-376.

Matérn, B. (2013). Spatial variation (Vol. 36). Springer Science \& Business Media.

McGraw, K. O., \& Wong, S. P. (1996). Forming inferences about some intraclass correlation coefficients. Psychological Methods, 1(1), 30-46.

Munoz, A., Carey, V., Schouten, J. P., Segal, M., \& Rosner, B. (1992). A parametric family of correlation structures for the analysis of longitudinal data. Biometrics, 733-742.

Newman, D. A., \& Sin, H.-P. (2020). Within-group agreement (r wg): Two theoretical parameters and their estimators. Organizational Research Methods, 23(1), 30-64.

Ramos-Villagrasa, P. J., Marques-Quinteiro, P., Navarro, J., \& Rico, R. (2018). Teams as complex adaptive systems: Reviewing 17 years of research. Small Group Research, 49(2), 135-176.

Rapp, T., Maynard, T., Domingo, M., \& Klock, E. (2021). Team emergent states: What has emerged in the literature over 20 years. Small Group Research, 52(1), 68-102.

Rasmussen, C. E. (2003). Gaussian processes in machine learning, In Summer school on machine learning. Springer.

Sherif, M. (1935). A study of some social factors in perception. Archives of Psychology, 27(187), $1-60$.

Shrout, P., \& Fleiss, J. (1979). Intraclass correlations: Uses in assessing rater reliability. Psychological Bulletin, 86(2), 420-428. 
Solinger, O. N., Jansen, P. G., \& Cornelissen, J. P. (2020). The emergence of moral leadership. Academy of Management Review, 45(3), 504-527.

Stein, M. L. (2012). Interpolation of spatial data: Some theory for kriging. Springer Science \& Business Media.

Stuart, A. M. (2010). Inverse problems: A bayesian perspective. Acta numerica, 19, 451-559.

Thornton, P. H., Ocasio, W., \& Lounsbury, M. (2012). The institutional logics perspective: A new approach to culture, structure and process. Oxford University Press.

Wang, M., Zhou, L., \& Zhang, Z. (2016). Dynamic modeling. Annual Review of Organizational Psychology and Organizational Behavior, 3(1), 241-266. https://doi.org/10.1146/annurevorgpsych-041015-062553

Wee, E. X. M., \& Taylor, M. S. (2018). Attention to change: A multilevel theory on the process of emergent continuous organizational change. Journal of Applied Psychology, 103(1), 1-13.

Wittmer, J. L., \& LeBreton, J. M. (2021). Interrater agreement and interrater reliability: Implications for multilevel research, In Oxford research encyclopedia of business and management.

Zaccaro, S. J., Dubrow, S., Torres, E. M., \& Campbell, L. N. (2020). Multiteam systems: An integrated review and comparison of different forms. Annual Review of Organizational Psychology and Organizational Behavior, 7, 479-503. 


\section{A ICC}

The most commonly used measure of consensus emergence is the intraclass correlation coefficient (ICC), originally introduced by Fisher (1932). There are many versions of ICC (Shrout \& Fleiss, 1979). McGraw and Wong (1996) gives a good overview for when the different versions are applicable. For ease of presentation, we limit our discussion to the most basic version of ICC, referred to as plain ICC, ICC1 (Bliese, 2000), ICC(1,1) (Shrout \& Fleiss, 1979) or ICC(1) (McGraw \& Wong, 1996). A formal definition of ICC follows.

Definition 2. Let $P_{i j}$ denote the quantity of individual $i=1, \ldots, n$ in a group $j$. The intraclass correlation coefficient, the correlation of two uniformly randomly selected individuals $i, k$ from group $j$, is

$$
I C C=\operatorname{Corr}\left[P_{i j}, P_{k j}\right]=\frac{\operatorname{Cov}\left[P_{i j}, P_{k j}\right]}{\sqrt{V\left[P_{i j}\right] V\left[P_{k j}\right]}} .
$$

More common to see when ICC is discussed in literature is the formula for calculation of the ICC in the multilevel setting of the variance components model (1):

$$
I C C=\frac{\sigma_{G}^{2}}{\sigma_{G}^{2}+\sigma_{P}^{2}},
$$

i.e., the proportion of variance in the outcome that is due to variation between groups.

One should note that this formula is only correct if the ICC is based on the variance components model (1), or equivalent, a one-way random effects model (Bartko, 1966 Goldstein, 2011). For an altered model, the ICC no longer simplifies to this expression.

\section{B The consensus emergence statistic $r(t)$}

In this section we first prove the expression for $r(t)$ for the models presented in the paper, and then derive a naive model free estimate of $r(t)$.

\section{B.1 Proofs of $r(t)$}

Here we give proofs of $r(t)$ for all presented models. Remember that we have $R_{t}=\mathbb{E}\left[\left(P_{i j}(t)-\right.\right.$ $\left.\left.P_{k j}(t)\right)^{2}\right]$, where $j$ is a uniform draw over all groups and $i$ and $k$ are uniform draws, without replacement, among individuals in group $j$.

Lemma 1. Assume we have the following model (Model 5),

$$
\begin{aligned}
P_{t i j} & =G_{j}(t)+e_{t i j}^{P}, \\
G_{j}(t) & =G_{0}+\exp \left(\delta_{G} t\right) e_{j}^{G},
\end{aligned}
$$

where $e_{t i j}^{P} \sim N\left(0, \sigma_{P}^{2} \exp \left[2 \delta_{P} t\right]\right)$, and $e_{j}^{G} \sim N\left(0, \sigma_{G}^{2}\right)$. Then

$$
r(t)=\exp \left[\delta_{P} t\right]
$$


Proof. We begin by finding $R_{t}$ for the model.

$$
\begin{aligned}
R_{t} & =\mathbb{E}\left[\left(P_{t i j}-P_{t k j}\right)^{2}\right] \\
& =\mathbb{E}\left[\left(G_{j}(t)+e_{t i j}^{P}-\left(G_{j}(t)+e_{t k j}^{P}\right)\right)^{2}\right] \\
& =\mathbb{E}\left[\left(e_{t i j}^{P}-e_{t k j}^{P}\right)^{2}\right] \\
& =\mathbb{E}\left[\left(e_{t i j}^{P}\right)^{2}\right]+\mathbb{E}\left[\left(e_{t k j}^{P}\right)^{2}\right]-2 \mathbb{E}\left[e_{t i j}^{P} e_{t k j}^{P}\right] \\
& =\mathbb{V}\left[e_{t i j}^{P}\right]+\mathbb{V}\left[e_{t k j}^{P}\right] \\
& =2 \sigma_{P}^{2} \exp \left[2 \delta_{P} t\right] .
\end{aligned}
$$

The second to last equality follows from that the error terms are assumed to be independent and identically distributed with expected value 0 . This gives

$$
\begin{aligned}
r(t) & =\sqrt{\frac{R_{t}}{R_{0}}} \\
& =\sqrt{\frac{2 \sigma_{Y}^{2} \exp \left[2 \delta_{Y} t\right]}{2 \sigma_{Y}^{2} \exp \left[2 \delta_{Y} * 0\right]}} \\
& =\sqrt{\exp \left[2 \delta_{Y} t\right]} \\
& =\sqrt{\exp \left[\delta_{Y} t\right]^{2}} \\
& =\exp \left[\delta_{Y} t\right] .
\end{aligned}
$$

Lemma 2. Assume we have the following Consensus Emergence Model:

$$
\begin{aligned}
P_{t i j} & =P_{i j}^{0}+G_{j}(t)+e_{t i j}^{P}, \\
e_{t i j}^{P} & \sim N\left(0, \sigma_{P}^{2} \exp \left[2 \delta_{1} t\right]\right), \\
P_{i j}^{0} & =e_{i j}^{P_{0}}, \\
e_{i j}^{P_{0}} & \sim N\left(0, \sigma_{P_{0}}^{2}\right), \\
G_{j}(t) & =\mu_{0}+t \mu_{1}+e_{j}^{G_{0}}+t e_{j}^{G_{1}}, \\
{\left[\begin{array}{c}
e_{j}^{G_{0}} \\
e_{j}^{G_{1}}
\end{array}\right] } & \sim N\left(\mathbf{0}, \boldsymbol{\Sigma}_{G}\right) .
\end{aligned}
$$

Then

$$
r(t)=\sqrt{\frac{\sigma_{P_{0}}^{2}+\sigma_{P}^{2} \exp \left[2 \delta_{1} t\right]}{\sigma_{P_{0}}^{2}+\sigma_{P}^{2}}} .
$$


Proof. We begin by finding $R_{t}$.

$$
\begin{aligned}
R_{t} & =\mathbb{E}\left[\left(P_{t i j}-P_{t k j}\right)^{2}\right] \\
& =\mathbb{E}\left[\left(P_{i j}^{0}+e_{t i j}^{P}-\left(P_{k j}^{0}+e_{t k j}^{P}\right)\right)^{2}\right] \\
& =\mathbb{E}\left[\left(e_{i j}^{P_{0}}+e_{t i j}^{P}-e_{k j}^{P_{0}}-e_{t k j}^{P}\right)^{2}\right] \\
& =\mathbb{E}\left[\left(e_{i j}^{P_{0}}\right)^{2}+\left(e_{t i j}^{P}\right)^{2}+\left(e_{k j}^{P_{0}}\right)^{2}+\left(e_{t k j}^{P}\right)^{2}\right] \\
& \left.=\mathbb{V}\left[e_{i j}^{P_{0}}\right)\right]+\mathbb{V}\left[e_{t i j}^{P}\right]+\mathbb{V}\left[e_{k j}^{P_{0}}\right]+\mathbb{V}\left[e_{t k j}^{P}\right] \\
& =2 \sigma_{P_{0}}^{2}+2 \sigma_{P}^{2} \exp \left[2 \delta_{1} t\right] .
\end{aligned}
$$

The fourth equality due to that all cross terms will eventually be zero, as all error terms are assumed to be independent of each other. This gives

$$
\begin{aligned}
r(t) & =\sqrt{\frac{R_{t}}{R_{0}}} \\
& =\sqrt{\frac{2 \sigma_{P_{0}}^{2}+2 \sigma_{P}^{2} \exp \left[2 \delta_{1} t\right]}{2 \sigma_{P_{0}}^{2}+2 \sigma_{P}^{2} \exp \left[2 \delta_{1} * 0\right]}} \\
& =\sqrt{\frac{\sigma_{P_{0}}^{2}+\sigma_{P}^{2} \exp \left[2 \delta_{1} t\right]}{\sigma_{P_{0}}^{2}+\sigma_{P}^{2}}} .
\end{aligned}
$$

Lemma 3. Assume we have the following Heterogeneous Consensus Emergence Model:

$$
\begin{aligned}
Y_{t i j} & =P_{t i j}+e_{t i j}^{Y}, \\
e_{t i j}^{Y} & \sim N\left(0, \sigma_{Y}^{2}\right), \\
P_{t i j} & =G_{j}(t)+e_{i j}^{P_{0}}+e_{t i j}^{P_{1}}, \\
e_{i j}^{P_{0}} & \sim N\left(0, \sigma_{P_{0}}^{2}\right), \\
e_{t i j}^{P_{1}} & \sim N\left(0, \sigma_{P_{1}}^{2} \exp \left[2 \delta_{1} t\right]\right), \\
G_{j}(t) & =\mu_{0}+t \mu_{1}+e_{j}^{G_{0}}+t e_{j}^{G_{1}}, \\
{\left[\begin{array}{c}
e_{j}^{G_{0}} \\
e_{j}^{G_{1}}
\end{array}\right] } & \sim N\left(\mathbf{0}, \boldsymbol{\Sigma}_{G}\right) .
\end{aligned}
$$

Then

$$
r(t)=\sqrt{\frac{\sigma_{P_{0}}^{2}+\sigma_{P_{1}}^{2} \exp \left[2 \delta_{1} t\right]}{\sigma_{P_{0}}^{2}+\sigma_{P_{1}}^{2}}} .
$$


Proof. Finding $R_{t}$ for the HetCEM is similar to finding it for the CEM.

$$
\begin{aligned}
R_{t} & =\mathbb{E}\left[\left(P_{t i j}-P_{t k j}\right)^{2}\right] \\
& =\mathbb{E}\left[\left(e_{i j}^{P_{0}}+e_{t i j}^{P_{1}}-\left(e_{k j}^{P_{0}}+e_{t k j}^{P_{1}}\right)\right)^{2}\right] \\
& =\mathbb{E}\left[\left(e_{i j}^{P_{0}}\right)^{2}+\left(e_{t i j}^{P_{1}}\right)^{2}+\left(e_{k j}^{P_{0}}\right)^{2}+\left(e_{t k j}^{P_{1}}\right)^{2}\right] \\
& \left.=\mathbb{V}\left[e_{i j}^{P_{0}}\right)\right]+\mathbb{V}\left[e_{t i j}^{P_{1}}\right]+\mathbb{V}\left[e_{k j}^{P_{0}}\right]+\mathbb{V}\left[e_{t k j}^{P_{1}}\right] \\
& =2 \sigma_{P_{0}}^{2}+2 \sigma_{P_{1}}^{2} \exp \left[2 \delta_{1} t\right] .
\end{aligned}
$$

This gives

$$
\begin{aligned}
r(t) & =\sqrt{\frac{R_{t}}{R_{0}}} \\
& =\sqrt{\frac{2 \sigma_{P_{0}}^{2}+2 \sigma_{P_{1}}^{2} \exp \left[2 \delta_{1} t\right]}{2 \sigma_{P_{0}}^{2}+2 \sigma_{P_{1}}^{2} \exp \left[2 \delta_{1} * 0\right]}} \\
& =\sqrt{\frac{\sigma_{P_{0}}^{2}+\sigma_{P_{1}}^{2} \exp \left[2 \delta_{1} t\right]}{\sigma_{P_{0}}^{2}+\sigma_{P_{1}}^{2}}} .
\end{aligned}
$$

Lemma 4. Assume we have the following Homogeneous Consensus Emergence Model

$$
\begin{aligned}
Y_{t i j} & =P_{i j}(t)+e_{t i j}^{Y}, \\
e_{t i j}^{Y} & \sim N\left(0, \sigma_{Y}^{2}\right), \\
P_{i j}(t) & =G_{j}(t)+e_{i j}^{P_{0}}+\exp \left[\delta_{1} t\right] e_{i j}^{P_{1}}, \\
e_{i j}^{P_{0}} & \sim N\left(0, \sigma_{P_{0}}^{2}\right), \\
e_{i j}^{P_{1}} & \sim N\left(0, \sigma_{P_{1}}^{2}\right), \\
G_{j}(t) & =\mu_{0}+t \mu_{1}+e_{j}^{G_{0}}+t e_{j}^{G_{1}}, \\
{\left[\begin{array}{c}
e_{j}^{G_{0}} \\
e_{j}^{G_{1}}
\end{array}\right] } & \sim N\left(\mathbf{0}, \boldsymbol{\Sigma}_{G}\right) .
\end{aligned}
$$

Then

$$
r(t)=\sqrt{\frac{\sigma_{P_{0}}^{2}+\sigma_{P_{1}}^{2} \exp \left[2 \delta_{1} t\right]}{\sigma_{P_{0}}^{2}+\sigma_{P_{1}}^{2}}} .
$$

Proof. We find $R_{t}$ for the HomCEM in the same way as for the HetCEM, with a slight alteration for the constant term $\exp \left[\delta_{1} t\right]$ multiplied with the $e^{P_{1}}$ error term. When taken out of the variance it is squared and $R_{t}$ becomes the same as for the HetCEM:

$$
R_{t}=2 \sigma_{P_{0}}^{2}+2 \sigma_{P_{1}}^{2} \exp \left[2 \delta_{1} t\right] .
$$


Thus the expression for $r(t)$ is also the same as for the HetCEM:

$$
r(t)=\sqrt{\frac{\sigma_{P_{0}}^{2}+\sigma_{P_{1}}^{2} \exp \left[2 \delta_{1} t\right]}{\sigma_{P_{0}}^{2}+\sigma_{P_{1}}^{2}}} .
$$

Lemma 5. Assume we have the following GP Consensus Emergence Model

$$
\begin{aligned}
Y_{t i j} & =P_{t i j}+e_{t i j}^{Y}, \\
e_{t i j}^{Y} & \sim N\left(0, \sigma_{Y}^{2}\right), \\
P_{t i j} & =G_{j}(t)+e_{i j}^{P_{0}}+e_{i j}^{P_{1}}(t), \\
e_{i j}^{P_{0}} & \sim N\left(0, \sigma_{P_{0}}^{2}\right), \\
e_{i j}^{P_{1}}(t) & \sim G P_{i j}(t), \\
G_{j}(t) & =\mu_{0}+t \mu_{1}+e_{j}^{G_{0}}+t e_{j}^{G_{1}}, \\
{\left[\begin{array}{c}
e_{j}^{G_{0}} \\
e_{j}^{G_{1}}
\end{array}\right] } & \sim N\left(\mathbf{0}, \boldsymbol{\Sigma}_{G}\right),
\end{aligned}
$$

where the $G P_{i j}(t)$ has exponential covariance function

$$
\Sigma\left(t_{1}, t_{2}\right)=\sigma_{P_{1}}^{2} \exp \left[\delta_{1}\left(t_{1}+t_{2}\right)\right] \exp \left(-\frac{\left|t_{1}-t_{2}\right|}{\kappa}\right) .
$$

Then

$$
r(t)=\sqrt{\frac{\sigma_{P_{0}}^{2}+\sigma_{P_{1}}^{2} \exp \left[2 \delta_{1} t\right]}{\sigma_{P_{0}}^{2}+\sigma_{P_{1}}^{2}}} .
$$

Proof. Similarly to finding $R_{t}$ for the HetCEM, we have

$$
\begin{aligned}
R_{t} & =\mathbb{E}\left[\left(P_{t i j}-P_{t k j}\right)^{2}\right] \\
& =\mathbb{E}\left[\left(e_{i j}^{P_{0}}+e_{i j}^{P_{1}}(t)-\left(e_{k j}^{P_{0}}+e_{k j}^{P_{1}}(t)\right)\right)^{2}\right] \\
& =\mathbb{E}\left[\left(e_{i j}^{P_{0}}\right)^{2}+\left(e_{i j}^{P_{1}}(t)\right)^{2}+\left(e_{k j}^{P_{0}}\right)^{2}+\left(e_{k j}^{P_{1}}(t)\right)^{2}\right] \\
& \left.=\mathbb{V}\left[e_{i j}^{P_{0}}\right)\right]+\mathbb{V}\left[e_{i j}^{P_{1}}(t)\right]+\mathbb{V}\left[e_{k j}^{P_{0}}\right]+\mathbb{V}\left[e_{k j}^{P_{1}}(t)\right] .
\end{aligned}
$$

To find the variances $\mathbb{V}\left[e_{i j}^{P_{1}}(t)\right]$ and $\mathbb{V}\left[e_{k j}^{P_{1}}(t)\right]$ we use the GP covariance function $\left[9\right.$, with $t_{1}=t_{2}=t$ :

$$
\begin{aligned}
\mathbb{V}\left[e_{i j}^{P_{1}}(t)\right] & =\Sigma(t, t) \\
& =\sigma_{P_{1}}^{2} \exp \left[\delta_{1}(2 t)\right] \exp \left(-\frac{|0|}{\kappa}\right) \\
& =\sigma_{P_{1}}^{2} \exp \left[2 \delta_{1} t\right] .
\end{aligned}
$$

The variances of $\mathbb{V}\left[e_{i j}^{P_{1}}(t)\right]$ and $\mathbb{V}\left[e_{k j}^{P_{1}}(t)\right]$ are the same as in the HetCEM and thus $R_{t}$ and $r(t)$ follows exactly as in the proof of Lemma 4 


\section{B.2 Empirical estimate of $r(t)$}

A naive model free estimate of $r(t)$ can be found by replacing $R_{t}$ with

$$
\hat{R}_{t}^{y}=\frac{1}{J} \sum_{j=1}^{J} \frac{1}{n_{j}\left(n_{j}-1\right)} \sum_{i=1}^{n_{j}} \sum_{k \neq i_{1}}\left(y_{i j t}-y_{k j t}\right)^{2},
$$

that is we use the data $y_{i j t}$ as an estimate of $P_{i j}(t)$. This gives

$$
\hat{r}^{y}(t)=\sqrt{\frac{\hat{R}_{t}^{Y}}{\hat{R}_{0}^{y}}}
$$

The issue of this statistic is that it is often biased. For instance in the regular three level model (2) then $\hat{r}(t)$ is an estimator of $\mathbb{E}_{y}\left[\hat{R}_{t}^{y}\right]=R_{t}+2 \sigma_{Y}^{2}$.

\section{Connection between GP and the HetCEM and HomCEM}

A very interesting property of using GP for consensus emergence is that if $\kappa$ is very large, in relation to the distance between the measurement locations $\left(t_{i}\right)$, this gives that the correlation between $G P$ at two measurement locations is zero and thus the model is reduced to the HetCEM. While if $\kappa$ is very small is very small in relation to the measurement locations the correlation between $G P$ at two measurement locations is, almost, one and thus the model is reduced to the HomCEM. In fact in the limit of the parameter range there is equality between the models which we prove in the appendix. Theoretically this is important for the HetCEM. The reason is that there exists no continuous processes that can have that form, i.e. $P\left(t_{1}\right)$ and $P\left(t_{2}\right)$ does not converge to the same value even when the difference between $t_{1}$ and $t_{2}$ goes to zero. But it is possible that one has a GP where the range is so small (with respect to the distance between measurement locations) that the correlation between measurement location can not be measured.

Below we prove the connection between the models.

\section{HetCEM and HomCEM as special cases of GP model}

We want to show that for the multilevel model using Gaussian processes and consensus emergence we can derive as a special case both the HomCEM and HetCEM. In order to do that we need to derive the distribution of the data under all models. Since in all three-level models the data $Y$ are a sum of Gaussian random variables we know that the distribution is Gaussian, and we need just establish the mean and covariance of the distributions.

Through out this section we assume that we have equal number of observations per individual and equal number of individuals per group. Thus we have $N M K$ observations where $N$ is the number of individual observations, $M$ number of individuals, and $K$ number of groups. In the proof we will column stack several random variables. We will always do this by the inner index to the outer index. This means that for instance the column stack observation vector is given by $\mathbf{Y}=\left[Y_{111}, Y_{211}, \ldots, Y_{121}, Y_{221}, \ldots, Y_{11 K}, \ldots, Y_{N M K}\right]^{T}$. We further assume that all individuals are observed at the same time points $\mathbf{t}_{P}=\left[t_{1}, \ldots, t_{N}\right]^{T}$, and we denote the corresponding to the vector $\mathbf{Y}$ as $\mathbf{t}$. The distribution of $\mathbf{Y}$ for all our model is given by

$$
\mathbf{Y} \sim \mathcal{N}(\boldsymbol{\mu}, \boldsymbol{\Sigma})
$$


where the mean and covariance can be decomposed into the three part, each defined by a level in the model. Before stating the result we need introduce some vector matrix notation we will use throughout the sections. We denote the unit column vector of length $m$ as $\mathbf{1}_{m}$, and the $N M \times 1$ vector $\mathbf{t}_{G}=\mathbf{t}_{P} \otimes \mathbf{1}_{M}$ where $\otimes$ is the Kronecker product.

We let $\mathbf{I}_{n}$ denote the identity matrix of size $n \times n$ and $\operatorname{Diag}(\mathbf{x})$ be the function that takes a vector $\mathbf{x}$, and returns a diagonal matrix with $\mathbf{x}$ on the diagonal.

$$
\begin{aligned}
& \boldsymbol{\mu}=\boldsymbol{\mu}_{O}+\boldsymbol{\mu}_{P}+\boldsymbol{\mu}_{G} \\
& \boldsymbol{\Sigma}=\boldsymbol{\Sigma}_{O}+\boldsymbol{\Sigma}_{P}+\boldsymbol{\Sigma}_{G} .
\end{aligned}
$$

Thus the each multilevel model is uniquely determined by the sum of the three vectors and matrices.

Lemma 6. Assume that $\mathbf{Y}$ is defined by the following three level multilevel model

$$
\begin{aligned}
Y_{t i j} & =P_{t i j}+e_{t i j}^{Y}, \\
e_{t i j}^{Y} & \sim N\left(0, \sigma_{Y}^{2}\right), \\
P_{t i j} & =G_{j}(t)+e_{i j}^{P_{0}}+e_{t i j}^{P_{1}}, \\
e_{i j}^{P_{0}} & \sim N\left(0, \sigma_{P_{0}}^{2}\right), \\
e_{t i j}^{P_{1}} & \sim N\left(0, \sigma_{P_{1}}^{2} \exp \left[2 \delta_{1} t\right]\right), \\
G_{j}(t) & =\mu_{0}+t \mu_{1}+e_{j}^{G_{0}}+t e_{j}^{G_{1}}, \\
{\left[\begin{array}{c}
e_{j}^{G_{0}} \\
e_{j}^{G_{1}}
\end{array}\right] } & \sim N\left(\mathbf{0}, \boldsymbol{\Sigma}_{G}\right) .
\end{aligned}
$$

Then

$$
\mathbf{Y} \sim \mathcal{N}\left(\boldsymbol{\mu}^{O}+\boldsymbol{\mu}^{P}+\boldsymbol{\mu}^{G}, \boldsymbol{\Sigma}^{O}+\boldsymbol{\Sigma}^{P}+\boldsymbol{\Sigma}^{G}\right) .
$$

Here the three mean and variance terms represents the contribution from each level and is given by

$$
\begin{aligned}
& \boldsymbol{\mu}^{O}=\mathbf{0}, \\
& \boldsymbol{\mu}^{P}=\mathbf{0}, \\
& \boldsymbol{\mu}^{G}=\mathbf{1} \mu_{0}+\mathbf{t} \mu_{1},
\end{aligned}
$$

and

$$
\begin{aligned}
& \boldsymbol{\Sigma}^{O}=\sigma_{e}^{2} \mathbf{I}_{N M K}, \\
& \boldsymbol{\Sigma}^{P}=\sigma_{p}^{2} \operatorname{Diag}\left(\exp \left[2 \delta_{1} \mathbf{t}\right]\right)+\sigma_{P_{0}}^{2}\left(\mathbf{I}_{M K} \otimes \mathbf{1}_{N} \mathbf{1}_{N}^{T}\right), \\
& \boldsymbol{\Sigma}^{G}=\mathbf{I}_{K} \otimes\left[\mathbf{1}_{N M}, \mathbf{t}_{G}\right] \boldsymbol{\Sigma}_{G}\left[\mathbf{1}_{N M}, \mathbf{t}_{G}\right]^{T} .
\end{aligned}
$$

Proof. Note that from definition $Y_{t i j}=e_{t i j}^{Y}+e_{i j}^{P_{0}}+e_{t i j}^{P_{1}}+\mu_{0}+t \mu_{1}+e_{j}^{G_{0}}+t e_{j}^{G_{1}}$ we will now derive the density of the column stacked the various noise components and from this derive the full density of $\mathbf{Y}$.

First let $\mathbf{e}^{Y}$ denote the column stacked $N M K \times 1$ vector of $e_{t i j}^{Y}$ which by definition $\mathbf{e}^{Y} \sim$ $\mathcal{N}\left(\mathbf{0}, \sigma_{Y}^{2} \mathbf{I}_{N M K}\right)$. The $N M K \times 1$ vector $\mathbf{e}^{P_{1}} \sim \mathcal{N}\left(\mathbf{0}, \sigma_{p}^{2} \operatorname{Diag}\left(\exp \left[2 \delta_{1} \mathbf{t}\right]\right)\right.$ and the $M K \times 1$ vector 
$\mathbf{e}^{P_{0}} \sim \mathcal{N}\left(\mathbf{0}, \sigma_{P_{0}}^{2} \mathbf{I}_{M K}\right)$. For the group noise, $e_{j}^{G_{0}}$ and $e_{j}^{G_{1}}$ we column stack hem jointly to a $2 K \times 1$ vector $\mathbf{e}^{G}=\left[e_{1}^{G_{0}}, e_{1}^{G_{1}}, e_{2}^{G_{0}}, e_{2}^{G_{1}}, \ldots, e_{K}^{G_{0}}, e_{K}^{G_{1}}\right]$, for which $\mathbf{e}^{G} \sim \mathcal{N}\left(\mathbf{0}, \mathbf{I}_{K} \otimes \mathbf{\Sigma}_{G}\right)$. Now we can add the noise plus the expectation term to get

$$
\mathbf{Y}=\mathbf{e}^{Y}+\mathbf{e}^{P_{1}}+\left(\mathbf{I}_{M K} \otimes \mathbf{1}_{N}\right) \mathbf{e}^{P_{0}}+\mathbf{1}_{N M K} \mu_{0}+\mathbf{t} \mu_{1}+\left(\mathbf{I}_{K} \otimes\left[\mathbf{1}_{N M}, \mathbf{t}_{G}\right]\right) \mathbf{e}^{G} .
$$

Thus $\boldsymbol{\mu}^{O}$ and $\boldsymbol{\Sigma}^{O}$ follows directly from the distribution of $\mathbf{e}^{Y}$. Clearly $\boldsymbol{\mu}^{P}$ is zero and the variance of $\mathbf{e}^{P_{1}}+\left(\mathbf{I}_{M K} \otimes \mathbf{1}_{N}\right) \mathbf{e}^{P_{0}}$ is given by

$$
\begin{aligned}
\boldsymbol{\Sigma}^{P} & =\mathbb{V}\left[\mathbf{e}^{P_{1}}\right]+\left(\mathbf{I}_{M K} \otimes \mathbf{1}_{N}\right) \mathbb{V}\left[\mathbf{e}^{P_{0}}\right]\left(\mathbf{I}_{M K} \otimes \mathbf{1}_{N}\right)^{T} \\
& =\sigma_{p}^{2} \operatorname{Diag}\left(\exp \left[2 \delta_{1} \mathbf{t}\right]\right)+\left(\mathbf{I}_{M K} \otimes \mathbf{1}_{N}\right) \sigma_{P_{0}}^{2} \mathbf{I}_{M K}\left(\mathbf{I}_{M K} \otimes \mathbf{1}_{N}\right)^{T} \\
& =\sigma_{p}^{2} \operatorname{Diag}\left(\exp \left[2 \delta_{1} \mathbf{t}\right]\right)+\sigma_{P_{0}}^{2}\left(\mathbf{I}_{M K} \otimes \mathbf{1}_{N} \mathbf{1}_{N}^{T}\right) .
\end{aligned}
$$

Finally, we have $\boldsymbol{\mu}^{G}=\mathbf{1} \mu_{0}+\mathbf{t} \mu_{1}$ and

$$
\begin{aligned}
\boldsymbol{\Sigma}^{G} & =\left(\mathbf{I}_{K} \otimes\left[\mathbf{1}_{N M}, \mathbf{t}_{G}\right]\right) \mathbb{V}\left[\mathbf{e}^{G}\right]\left(\mathbf{I}_{K} \otimes\left[\mathbf{1}_{N M}, \mathbf{t}_{G}\right]\right)^{T} \\
& =\left(\mathbf{I}_{K} \otimes\left[\mathbf{1}_{N M}, \mathbf{t}_{G}\right]\right)\left(\mathbf{I}_{K} \otimes \boldsymbol{\Sigma}_{G}\right)\left(\mathbf{I}_{K} \otimes\left[\mathbf{1}_{N M}, \mathbf{t}_{G}\right]\right)^{T} \\
& =\mathbf{I}_{K} \otimes\left[\mathbf{1}_{N M}, \mathbf{t}_{G}\right] \boldsymbol{\Sigma}_{G}\left[\mathbf{1}_{N M}, \mathbf{t}_{G}\right]^{T} .
\end{aligned}
$$

Lemma 7. Assume that $\mathbf{Y}$ is defined by the following three level multilevel model

$$
\begin{aligned}
Y_{t i j} & =P_{i j}(t)+e_{t i j}^{Y}, \\
e_{t i j}^{Y} & \sim N\left(0, \sigma_{Y}^{2}\right), \\
P_{i j}(t) & =G_{j}(t)+e_{i j}^{P_{0}}+\exp \left[\delta_{1} t\right] e_{i j}^{P_{1}}, \\
e_{i j}^{P_{0}} & \sim N\left(0, \sigma_{P_{0}}^{2}\right), \\
e_{i j}^{P_{1}} & \sim N\left(0, \sigma_{P_{1}}^{2}\right), \\
G_{j}(t) & =\mu_{0}+t \mu_{1}+e_{j}^{G_{0}}+t e_{j}^{G_{1}}, \\
{\left[\begin{array}{c}
e_{j}^{G_{0}} \\
e_{j}^{G_{1}}
\end{array}\right] } & \sim N\left(\mathbf{0}, \boldsymbol{\Sigma}_{G}\right) .
\end{aligned}
$$

Then

$$
\mathbf{Y} \sim \mathcal{N}\left(\boldsymbol{\mu}^{O}+\boldsymbol{\mu}^{P}+\boldsymbol{\mu}^{G}, \boldsymbol{\Sigma}^{O}+\boldsymbol{\Sigma}^{P}+\boldsymbol{\Sigma}^{G}\right)
$$

Here the three mean and variance terms represents the contribution from each level and is given by

$$
\begin{aligned}
& \boldsymbol{\mu}^{O}=\mathbf{0}, \\
& \boldsymbol{\mu}^{P}=\mathbf{0}, \\
& \boldsymbol{\mu}^{G}=\mathbf{1} \mu_{0}+\mathbf{t} \mu_{1},
\end{aligned}
$$

and

$$
\begin{aligned}
& \boldsymbol{\Sigma}^{O}=\sigma_{Y}^{2} \mathbf{I}_{N M K}, \\
& \boldsymbol{\Sigma}^{P}=\sigma_{P_{0}}^{2}\left(\mathbf{I}_{M K} \otimes \mathbf{1}_{N}^{T} \mathbf{1}_{N}\right)+\sigma_{P_{1}}^{2}\left(\mathbf{I}_{M K} \otimes \exp \left[\delta_{1} \mathbf{t}_{P}^{T}\right]\left(\exp \left[\delta_{1} \mathbf{t}_{P}^{T}\right]\right)^{T}\right), \\
& \boldsymbol{\Sigma}^{G}=\mathbf{I}_{K} \otimes\left[\mathbf{1}_{N M}^{T}, \mathbf{t}_{G}^{T}\right] \boldsymbol{\Sigma}_{G}\left[\mathbf{1}_{N M}^{T}, \mathbf{t}_{G}^{T}\right]^{T} .
\end{aligned}
$$


Proof. The proof is identical to the proof of Lemma 6 except for the term $\mathbf{e}^{P_{1}}$, which here is an $M K \times 1$ vector, $\mathbf{e}^{P_{1}} \sim \mathcal{N}\left(\mathbf{0}, \sigma_{P_{1}}^{2} \mathbf{I}_{M K}\right)$. Thus we only need to show that the variance of $\left(\mathbf{I}_{M K} \otimes \exp \left[\delta_{1} \mathbf{t}_{P}\right]\right) \mathbf{e}^{P_{1}}$ is $\sigma_{P_{1}}^{2}\left(\mathbf{I}_{M K} \otimes \exp \left[\delta_{1} \mathbf{t}_{P}\right]\left(\exp \left[\delta_{1} \mathbf{t}_{P}\right]\right)^{T}\right)$.

The variance of $\left(\mathbf{I}_{M K} \otimes \exp \left[\delta_{1} \mathbf{t}_{P}\right]\right) \mathbf{e}^{P_{1}}$ is given by

$$
\begin{aligned}
\mathbb{V}\left[\left(\mathbf{I}_{M K} \otimes \exp \left[\delta_{1} \mathbf{t}_{P}\right]\right) \mathbf{e}^{P_{1}}\right] & =\left(\mathbf{I}_{M K} \otimes \exp \left[\delta_{1} \mathbf{t}_{P}\right]\right) \mathbb{V}\left[\mathbf{e}^{P_{1}}\right]\left(\mathbf{I}_{M K} \otimes \exp \left[\delta_{1} \mathbf{t}_{P}\right]\right)^{T} \\
& =\left(\mathbf{I}_{M K} \otimes \exp \left[\delta_{1} \mathbf{t}_{P}\right]\right) \sigma_{P_{1}}^{2} \mathbf{I}_{M K}\left(\mathbf{I}_{M K} \otimes \exp \left[\delta_{1} \mathbf{t}_{P}\right]\right)^{T} \\
& =\sigma_{P_{1}}^{2}\left(\mathbf{I}_{M K} \otimes \exp \left[\delta_{1} \mathbf{t}_{P}\right]\left(\exp \left[\delta_{1} \mathbf{t}_{P}\right]\right)^{T}\right) .
\end{aligned}
$$

Lemma 8. Assume that $\mathbf{Y}$ is defined by the following three level multilevel model

$$
\begin{aligned}
Y_{t i j} & =P_{i j}(t)+e_{t i j}^{Y}, \\
e_{t i j}^{Y} & \sim N\left(0, \sigma_{Y}^{2}\right), \\
P_{i j}(t) & =G_{j}(t)+e_{i j}^{P_{0}}+e_{i j}^{P_{1}}(t), \\
e_{i j}^{P_{0}} & \sim N\left(0, \sigma_{P_{0}}^{2}\right), \\
e_{i j}^{P_{1}}(t) & \sim G P_{i j}(t), \\
G_{j}(t) & =\mu_{0}+t \mu_{1}+e_{j}^{G_{0}}+t e_{j}^{G_{1}}, \\
{\left[\begin{array}{c}
e_{j}^{G_{0}} \\
e_{j}^{G_{1}}
\end{array}\right] } & \sim N\left(\mathbf{0}, \boldsymbol{\Sigma}_{G}\right),
\end{aligned}
$$

where $G P_{i j}(t)$ is a Gaussian processes with covariance function

$$
r\left(t_{1}, t_{2}\right)=\sigma^{2} \exp \left(\delta\left(t_{1}+t_{2}\right)\right) \exp \left(-\frac{\left|t_{1}-t_{2}\right|}{\kappa}\right) .
$$

Then

$$
\mathbf{Y} \sim \mathcal{N}\left(\boldsymbol{\mu}^{O}+\boldsymbol{\mu}^{P}+\boldsymbol{\mu}^{G}, \boldsymbol{\Sigma}^{O}+\boldsymbol{\Sigma}^{P}+\boldsymbol{\Sigma}^{G}\right) .
$$

Here the three mean and variance terms represents the contribution from each level and is given by

$$
\begin{aligned}
\boldsymbol{\mu}^{O} & =\mathbf{0}, \\
\boldsymbol{\mu}^{P} & =\mathbf{0}, \\
\boldsymbol{\mu}^{G} & =\mathbf{1} \mu_{0}+\mathbf{t} \mu_{1},
\end{aligned}
$$

and

$$
\begin{aligned}
& \boldsymbol{\Sigma}^{O}=\sigma_{e}^{2} \mathbf{I}_{N M K}, \\
& \boldsymbol{\Sigma}^{P}=\left(\mathbf{I}_{M K} \otimes \boldsymbol{\Sigma}^{G P}\right)+\sigma_{P_{0}}^{2}\left(\mathbf{I}_{M K} \otimes \mathbf{1}_{N} \mathbf{1}_{N}^{T}\right), \\
& \boldsymbol{\Sigma}^{G}=\mathbf{I}_{K} \otimes\left[\mathbf{1}_{N M}, \mathbf{t}_{G}\right] \boldsymbol{\Sigma}_{G}\left[\mathbf{1}_{N M}, \mathbf{t}_{G}\right]^{T},
\end{aligned}
$$

where $\boldsymbol{\Sigma}^{G P}$ is a $N \times N$ matrix with $\Sigma^{G P}\left(t_{i}, t_{j}\right)=r\left(t_{i}, t_{j}\right)$. 
Proof. The proof is identical to the proof of Lemma 6 with the exception of the $\mathbf{e}^{P_{1}}$ term which now is a column stacked vector of observations of Gaussian processes instead. Thus we need only to show that $\mathbf{e}^{P_{1}}$ has variance $\left(\mathbf{I}_{M K} \otimes \boldsymbol{\Sigma}^{G P}\right)$, however this is follows immediately from that $\mathbf{e}^{P_{1}}$ are $M K$ column stacked independent $N$ dimensional Gaussian random variables with variance $\boldsymbol{\Sigma}^{G P}$.

Lemma 9. The Gaussian distributions defined in Lemma 6 and Lemma 6 are special cases of the Gaussian distribution in Lemma 8 .

Proof. From the distribution $\mathbf{Y}$ for HetCEM and the Gaussian processes multilevel models it is clear that it suffices to show that there exists a vector of $\left(\sigma^{2}, \delta, \kappa\right)$ such that $\boldsymbol{\Sigma}^{G P}$ equals $\sigma_{p}^{2} \operatorname{Diag}\left(\exp \left[2 \delta_{1} \mathbf{t}\right]\right)$. This can obtain be obtained by setting $\left(\sigma^{2}, \delta, \kappa\right)=\left(\sigma_{p}^{2}, \delta_{1}, 0\right)$ since in this case

$$
\begin{aligned}
\Sigma_{i j}^{G P} & = \begin{cases}\sigma_{p}^{2} \exp \left(2 \delta_{1} t_{i}\right) \exp (0) & \text { if } i=j, \\
\sigma_{p}^{2} \exp \left(\delta_{1} t_{i}+t_{j}\right) \exp (-\infty) & \text { if } i \neq j,\end{cases} \\
& = \begin{cases}\sigma_{p}^{2} \exp \left(2 \delta_{1} t_{i}\right) & \text { if } i=j, \\
0 & \text { if } i \neq j .\end{cases}
\end{aligned}
$$

Similarly we obtain HomCEM covariance by setting $\left(\sigma^{2}, \delta, \kappa\right)=\left(\sigma_{p}^{2}, \delta_{1}, \infty\right)$

$$
\begin{aligned}
\Sigma_{i j}^{G P} & = \begin{cases}\sigma_{p}^{2} \exp \left(2 \delta_{1} t_{i}\right) \exp (0) & \text { if } i=j, \\
\sigma_{p}^{2} \exp \left(\delta_{1} t_{i}+t_{j}\right) \exp (0) & \text { if } i \neq j,\end{cases} \\
& = \begin{cases}\sigma_{p}^{2} \exp \left(2 \delta_{1} t_{i}\right) & \text { if } i=j \\
\sigma_{p}^{2} \exp \left(\delta_{1} t_{i}+t_{j}\right) & \text { if } i \neq j\end{cases}
\end{aligned}
$$




\section{Simulation Results}

Detailed results of the simulations detailed in Section 7.2 are provided in Tables 447 . Details and results of the additional simulation of homogeneous consensus emergence are reported in the following section.

Table 4: Results HetCE, median values $\left(\left(p_{66}-p_{33}\right) / 2\right)$

\begin{tabular}{lcrrrrr}
\hline Model & $\delta_{1}$ & $r\left(t_{\max }\right)$ & $\hat{\delta_{1}}$ & $\hat{\sigma}_{P_{1}}^{2}$ & $\hat{r}\left(t_{\max }\right)$ & $\% \mathrm{NA}$ \\
\hline HetCEM & -0.03 & 0.92 & $-0.05(0.07)$ & $0.06(0.03)$ & $0.93(0.04)$ & 0 \\
HomCEM & & & $-0.04(0.11)$ & $0.04(0.03)$ & $0.97(0.06)$ & 0 \\
GP & & & $-0.10(0.16)$ & $0.05(0.02)$ & $0.90(0.04)$ & 0 \\
HetCEM GP & & & $-0.05(0.09)$ & $0.08(0.03)$ & $0.90(0.06)$ & 0.1 \\
HomCEM GP & & & $-0.30(0.34)$ & $0.02(0.03)$ & $0.98(0.08)$ & 0 \\
GP GP & & & $-0.07(0.12)$ & $0.07(0.02)$ & $0.90(0.06)$ & 0 \\
\hline HetCEM & -0.05 & 0.88 & $-0.09(0.11)$ & $0.07(0.03)$ & $0.89(0.04)$ & 0 \\
HomCEM & & & $-0.06(0.16)$ & $0.05(0.02)$ & $0.93(0.07)$ & 0 \\
GP & & $-0.12(0.14)$ & $0.06(0.02)$ & $0.87(0.04)$ & 0 \\
HetCEM GP & & & $-0.08(0.09)$ & $0.10(0.03)$ & $0.87(0.05)$ & 0 \\
HomCEM GP & & & $-0.41(0.36)$ & $0.04(0.03)$ & $0.91(0.09)$ & 0 \\
GP GP & & $-0.10(0.11)$ & $0.08(0.03)$ & $0.85(0.05)$ & 0.2 \\
\hline HetCEM & -0.08 & 0.83 & $-0.13(0.13)$ & $0.08(0.03)$ & $0.85(0.03)$ & 0 \\
HomCEM & & & $-0.11(0.29)$ & $0.06(0.02)$ & $0.88(0.07)$ & 0 \\
GP & & $-0.12(0.10)$ & $0.09(0.02)$ & $0.83(0.04)$ & 0.2 \\
HetCEM GP & & $-0.12(0.09)$ & $0.11(0.03)$ & $0.82(0.04)$ & 0 \\
HomCEM GP & & $-0.54(0.49)$ & $0.05(0.03)$ & $0.85(0.12)$ & 0 \\
GP GP & & $-0.14(0.11)$ & $0.10(0.02)$ & $0.81(0.04)$ & 0 \\
\hline HetCEM & 0 & 1 & $-0.01(0.03)$ & $0.06(0.03)$ & $1.00(0.03)$ & 0.1 \\
HomCEM & & & $-0.03(0.14)$ & $0.03(0.03)$ & $1.00(0.03)$ & 0 \\
GP & & $-0.01(0.07)$ & $0.02(0.02)$ & $1.00(0.04)$ & 0.3 \\
HetCEM GP & & $-0.01(0.02)$ & $0.07(0.03)$ & $0.99(0.04)$ & 0.1 \\
HomCEM GP & & & $-0.13(0.35)$ & $0.01(0.02)$ & $1.00(0.09)$ & 0.1 \\
GP GP & & & $0.01(0.06)$ & $0.03(0.03)$ & $1.00(0.07)$ & 0.6 \\
\hline
\end{tabular}


Table 5: Results HomCE, median values $\left(\left(p_{66}-p_{33}\right) / 2\right)$

\begin{tabular}{lcrrrrr}
\hline Model & $\delta_{1}$ & $r\left(t_{\max }\right)$ & $\hat{\delta}_{1}$ & $\hat{\sigma}_{P_{1}}^{2}$ & $\hat{r}\left(t_{\max }\right)$ & $\%$ NA \\
\hline HetCEM & & & $-0.01(0.02)$ & $0.04(0.02)$ & $1.00(0.01)$ & 0.1 \\
HomCEM & -0.03 & 0.92 & $-0.03(0.02)$ & $0.10(0.06)$ & $0.94(0.04)$ & 0 \\
GP & & & $-0.03(0.11)$ & $0.02(0.01)$ & $1.00(0.01)$ & 0 \\
HetCEM GP & & & $-0.03(0.14)$ & $0.04(0.02)$ & $0.98(0.03)$ & 0 \\
HomCEM GP & & & $-0.20(0.15)$ & $0.05(0.04)$ & $0.91(0.07)$ & 0.1 \\
GP GP & & & $-0.01(0.15)$ & $0.01(0.02)$ & $1.00(0.01)$ & 0.3 \\
\hline HetCEM & & $-0.02(0.02)$ & $0.04(0.02)$ & $0.99(0.01)$ & 0.1 \\
HomCEM & -0.05 & \multirow{2}{*}{0.88} & $-0.04(0.03)$ & $0.12(0.05)$ & $0.89(0.04)$ & 0 \\
GP & & $-0.04(0.14)$ & $0.02(0.01)$ & $0.99(0.02)$ & 0 \\
HetCEM GP & & & $-0.04(0.16)$ & $0.04(0.02)$ & $0.97(0.03)$ & 0 \\
HomCEM GP & & & $-0.23(0.12)$ & $0.07(0.03)$ & $0.86(0.06)$ & 0 \\
GP GP & & $-0.01(0.15)$ & $0.01(0.02)$ & $1.00(0.02)$ & 0.2 \\
\hline HetCEM & & & $-0.02(0.03)$ & $0.03(0.02)$ & $0.98(0.02)$ & 0 \\
HomCEM & -0.08 & 0.83 & $-0.05(0.03)$ & $0.15(0.04)$ & $0.84(0.04)$ & 0 \\
GP & & $-0.05(0.16)$ & $0.02(0.01)$ & $0.98(0.02)$ & 0 \\
HetCEM GP & & & $-0.05(0.22)$ & $0.04(0.01)$ & $0.96(0.03)$ & 0 \\
HomCEM GP & & & $-0.26(0.06)$ & $0.10(0.03)$ & $0.80(0.05)$ & 0 \\
GP GP & & $-0.03(0.14)$ & $0.01(0.02)$ & $1.00(0.03)$ & 0.3 \\
\hline HetCEM & & $-0.01(0.02)$ & $0.04(0.02)$ & $1.00(0.01)$ & 0 \\
HomCEM & 0 & 1 & $-0.91(0.02)$ & $0.05(0.05)$ & $1.00(0.02)$ & 0 \\
GP & & $-0.03(0.10)$ & $0.01(0.01)$ & $1.00(0.01)$ & 0 \\
HetCEM GP & & & $-0.02(0.04)$ & $0.04(0.02)$ & $0.99(0.02)$ & 0 \\
HomCEM GP & & & $0.01(0.25)$ & $0.02(0.02)$ & $1.00(0.08)$ & 0.1 \\
GP GP & & & $0.00(0.17)$ & $0.01(0.01)$ & $1.00(0.01)$ & 0.4 \\
\hline
\end{tabular}


Table 6: Results HetCE + GP, median values $\left(\left(p_{66}-p_{33}\right) / 2\right)$

\begin{tabular}{lcrrrrr}
\hline Model & $\delta_{1}$ & $r\left(t_{\max }\right)$ & $\hat{\delta}_{1}$ & $\hat{\sigma}_{P_{1}}^{2}$ & $\hat{r}\left(t_{\max }\right)$ & $\%$ NA \\
\hline HetCEM & & & $-0.02(0.04)$ & $0.06(0.04)$ & $0.98(0.04)$ & 0.1 \\
HomCEM & & & $-0.05(0.17)$ & $0.03(0.03)$ & $1.00(0.04)$ & 0 \\
GP & & $-0.02(0.04)$ & $0.03(0.03)$ & $0.99(0.04)$ & 0.1 \\
HetCEM GP & -0.03 & 0.92 & $-0.04(0.03)$ & $0.09(0.03)$ & $0.91(0.04)$ & 0 \\
HomCEM GP & & & $-0.22(0.60)$ & $0.02(0.02)$ & $1.00(0.06)$ & 0.1 \\
GP GP & & & $-0.06(0.09)$ & $0.07(0.03)$ & $0.90(0.04)$ & 0.2 \\
\hline HetCEM & & $-0.04(0.05)$ & $0.07(0.04)$ & $0.93(0.05)$ & 0 \\
HomCEM & & $-0.05(0.18)$ & $0.03(0.03)$ & $0.98(0.05)$ & 0 \\
GP & & $-0.04(0.07)$ & $0.05(0.03)$ & $0.92(0.05)$ & 0 \\
HetCEM GP & -0.05 & 0.88 & $-0.05(0.05)$ & $0.10(0.03)$ & $0.87(0.04)$ & 0 \\
HomCEM GP & & & $-0.30(0.69)$ & $0.03(0.03)$ & $0.95(0.08)$ & 0 \\
GP GP & & $-0.07(0.08)$ & $0.08(0.03)$ & $0.86(0.04)$ & 0.2 \\
\hline HetCEM & & & $-0.07(0.07)$ & $0.09(0.04)$ & $0.89(0.04)$ & 0 \\
HomCEM & & $-0.06(0.20)$ & $0.04(0.03)$ & $0.96(0.06)$ & 0 \\
GP & & $-0.06(0.10)$ & $0.08(0.03)$ & $0.87(0.04)$ & 0.2 \\
HetCEM GP & -0.08 & 0.83 & $-0.08(0.06)$ & $0.11(0.03)$ & $0.83(0.04)$ & 0 \\
HomCEM GP & & $-0.64(0.97)$ & $0.05(0.02)$ & $0.87(0.09)$ & 0 \\
GP GP & & $-0.08(0.07)$ & $0.11(0.03)$ & $0.83(0.03)$ & 0.3 \\
\hline HetCEM & & & $0.01(0.02)$ & $0.05(0.04)$ & $1.01(0.03)$ & 0.3 \\
HomCEM & & & $-0.03(0.09)$ & $0.02(0.03)$ & $1.00(0.01)$ & 0 \\
GP & & $0.02(0.03)$ & $0.03(0.03)$ & $1.03(0.05)$ & 0.3 \\
HetCEM GP & 0 & 1 & $0.00(0.02)$ & $0.09(0.03)$ & $1.00(0.04)$ & 0 \\
HomCEM GP & & & $-0.05(0.34)$ & $0.00(0.02)$ & $1.00(0.03)$ & 0 \\
GP GP & & & $-0.02(0.07)$ & $0.03(0.02)$ & $1.00(0.05)$ & 0.8 \\
\hline
\end{tabular}


Table 7: Results HomCE + GP, median values $\left(\left(p_{66}-p_{33}\right) / 2\right)$

\begin{tabular}{lcrrrrr}
\hline Model & $\delta_{1}$ & $r\left(t_{\max }\right)$ & $\hat{\delta}_{1}$ & $\hat{\sigma}_{P_{1}}^{2}$ & $\hat{r}\left(t_{\max }\right)$ & $\%$ NA \\
\hline HetCEM & & $0.01(0.03)$ & $0.02(0.02)$ & $1.01(0.02)$ & 0 \\
HomCEM & & $-0.02(0.01)$ & $0.13(0.07)$ & $0.96(0.04)$ & 0.1 \\
GP & & $0.02(0.02)$ & $0.03(0.02)$ & $1.02(0.02)$ & 0.2 \\
HetCEM GP & & & $-0.02(0.02)$ & $0.04(0.01)$ & $0.99(0.01)$ & 0 \\
HomCEM GP & -0.03 & 0.92 & $-0.04(0.07)$ & $0.05(0.04)$ & $0.94(0.05)$ & 0 \\
GP GP & & & $-0.12(0.43)$ & $0.01(0.01)$ & $0.99(0.02)$ & 0 \\
\hline HetCEM & & $0.01(0.03)$ & $0.03(0.02)$ & $1.00(0.02)$ & 0 \\
HomCEM & & $-0.03(0.01)$ & $0.14(0.06)$ & $0.92(0.05)$ & 0 \\
GP & & $0.02(0.03)$ & $0.03(0.02)$ & $1.02(0.02)$ & 0.3 \\
HetCEM GP & & $-0.02(0.03)$ & $0.04(0.02)$ & $0.99(0.02)$ & 0 \\
HomCEM GP & -0.05 & \multirow{2}{*}{0.88} & $-0.05(0.05)$ & $0.07(0.04)$ & $0.90(0.04)$ & 0 \\
GP GP & & $-0.08(0.05)$ & $0.02(0.02)$ & $0.98(0.02)$ & 0 \\
\hline HetCEM & & & $0.00(0.03)$ & $0.03(0.02)$ & $1.00(0.02)$ & 0 \\
HomCEM & & $-0.04(0.01)$ & $0.15(0.05)$ & $0.86(0.05)$ & 0.1 \\
GP & & $0.01(0.03)$ & $0.03(0.02)$ & $1.01(0.03)$ & 0.2 \\
HetCEM GP & & $-0.03(0.06)$ & $0.04(0.02)$ & $0.98(0.02)$ & 0 \\
HomCEM GP & -0.08 & 0.83 & $-0.09(0.06)$ & $0.09(0.04)$ & $0.85(0.04)$ & 0 \\
GP GP & & $-0.13(0.20)$ & $0.03(0.02)$ & $0.96(0.04)$ & 0.1 \\
\hline HetCEM & & & $0.02(0.03)$ & $0.03(0.02)$ & $1.01(0.02)$ & 0.1 \\
HomCEM & & & $0.00(0.01)$ & $0.09(0.07)$ & $1.00(0.01)$ & 0.3 \\
GP & & $0.02(0.02)$ & $0.03(0.02)$ & $1.03(0.03)$ & 0.1 \\
HetCEM GP & & & $-0.01(0.02)$ & $0.04(0.02)$ & $1.00(0.01)$ & 0 \\
HomCEM GP & 0 & 1 & $-0.01(0.07)$ & $0.02(0.03)$ & $1.00(0.01)$ & 0 \\
GP GP & & $-0.08(0.49)$ & $0.02(0.03)$ & $1.00(0.02)$ & 0.2 \\
\hline
\end{tabular}

\section{Simulation: strong consensus emergence effect}

We perform an additional simulation for homogeneous consensus emergence where we simulate data from a HomCEM model. To generate a $r\left(t_{\max }\right)$ value of 0.1 , we use $\sigma_{P_{0}}^{2}=0.001$ and $\delta_{1}=-0.7$. All other parameters are the same as in the simulations of regular models given in Table 3 . Results are given in Table 8

Table 8: Results from simulation of homogeneous consensus emergence with an $r\left(t_{\max }\right)$ value of 0.10. Reported values are median values of 1000 repetitions, together with a robust estimate of the standard deviation: $\left(p_{66}-p_{33}\right) / 2$ (in parenthesis).

\begin{tabular}{lcrrrrr}
\hline Model & $\delta_{1}$ & $r\left(t_{\max }\right)$ & $\hat{\delta}_{1}$ & $\hat{\sigma}_{P_{1}}^{2}$ & $\hat{r}\left(t_{\max }\right)$ & $\%$ NA \\
\hline HetCEM & & & $-0.63(0.27)$ & $0.09(0.01)$ & $0.26(0.06)$ & 0 \\
HomCEM & -0.7 & 0.10 & $-0.72(0.08)$ & $0.10(0.01)$ & $0.06(0.04)$ & 0 \\
GP & & & $-0.77(0.18)$ & $0.09(0.01)$ & $0.23(0.03)$ & 0 \\
HetCEM GP & & & $-0.73(0.17)$ & $0.11(0.01)$ & $0.21(0.04)$ & 0 \\
HomCEM GP & & $-0.70(0.06)$ & $0.14(0.02)$ & $0.03(0.02)$ & 0 \\
GP GP & & & $-0.73(0.12)$ & $0.11(0.02)$ & $0.17(0.06)$ & 0.4 \\
\hline
\end{tabular}

